\title{
A FOXO-Pak1 transcriptional pathway controls neuronal polarity
}

\author{
Luis de la Torre-Ubieta, ${ }^{1,2}$ Brice Gaudillière, ${ }^{1}$ Yue Yang, ${ }^{1,2,5}$ Yoshiho Ikeuchi, ${ }^{1,5}$ Tomoko Yamada, ${ }^{1,5}$ \\ Sara DiBacco, ${ }^{1}$ Judith Stegmüller, ${ }^{1,6}$ Ulrich Schüller, ${ }^{3,7}$ Dervis A. Salih, ${ }^{4}$ David Rowitch, ${ }^{3,8}$ \\ Anne Brunet, ${ }^{4}$ and Azad Bonni ${ }^{1,2,9}$ \\ ${ }^{1}$ Department of Pathology, Harvard Medical School, Boston, Massachusetts 02115, USA; ${ }^{2}$ Program in Neuroscience, Harvard \\ Medical School, Boston, Massachusetts 02115, USA; ${ }^{3}$ Department of Pediatric Oncology, Dana-Farber Cancer Institute, \\ Harvard Medical School, Boston, Massachusetts 02115, USA; ${ }^{4}$ Department of Genetics, Stanford University, Stanford, \\ California 94305, USA
}

\begin{abstract}
Neuronal polarity is essential for normal brain development and function. However, cell-intrinsic mechanisms that govern the establishment of neuronal polarity remain to be identified. Here, we report that knockdown of endogenous FOXO proteins in hippocampal and cerebellar granule neurons, including in the rat cerebellar cortex in vivo, reveals a requirement for the FOXO transcription factors in the establishment of neuronal polarity. The FOXO transcription factors, including the brain-enriched protein FOXO6, play a critical role in axo-dendritic polarization of undifferentiated neurites, and hence in a switch from unpolarized to polarized neuronal morphology. We also identify the gene encoding the protein kinase Pak1, which acts locally in neuronal processes to induce polarity, as a critical direct target gene of the FOXO transcription factors. Knockdown of endogenous Pak1 phenocopies the effect of FOXO knockdown on neuronal polarity. Importantly, exogenous expression of Pak1 in the background of FOXO knockdown in both primary neurons and postnatal rat pups in vivo restores the polarized morphology of neurons. These findings define the FOXO proteins and Pak1 as components of a cell-intrinsic transcriptional pathway that orchestrates neuronal polarity, thus identifying a novel function for the FOXO transcription factors in a unique aspect of neural development.
\end{abstract}

[Keywords: FOXO; neuronal polarity; Pak1; transcription; axons; dendrites]

Supplemental material is available at http://www.genesdev.org.

Received October 30, 2009; revised version accepted February 26, 2010.

Axo-dendritic polarity is a fundamental property of neurons that is essential for the establishment of proper neuronal connectivity, and provides the basis for directional flow of information in the nervous system (Ramón y Cajal 1995; Kandel et al. 2000). Neuronal polarity arises from the specification of undifferentiated neurites into axons and dendrites followed by their coordinate growth, leading to a neuronal shape typically with a long axon and several shorter dendrites. A major goal in neurobiology is to elucidate the mechanisms that govern the establishment of neuronal polarity. Biochemical events that act locally within neuronal processes leading to neuronal polarity have been characterized (Craig and Banker 1994;

\footnotetext{
${ }^{5}$ These authors contributed equally to this work.

Present addresses: ${ }^{6}$ Max-Planck-Institute of Experimental Medicine, Hermann-Rein-Str. 3, 37075 Göttingen, Germany; ${ }^{7}$ Center for Neuropathology and Prion Research, Ludwig-Maximilians-Universität, FeodorLynen-St 23, 81377 Munich, Germany; ${ }^{8}$ Department of Pediatrics, Department of Neurological Surgery, and Howard Hughes Medical Institute, University of California at San Francisco, San Francisco, CA 94143, USA. ${ }^{9}$ Corresponding author.

E-MAIL azad_bonni@hms.harvard.edu; FAX (617) 432-4101.

Supplemental material is available at http://www.genesdev.org.

Article is online at http://www.genesdev.org/cgi/doi/10.1101/gad.1880510.
}

Jan and Jan 2003; Shi et al. 2003; Schwamborn and Puschel 2004; de Anda et al. 2005; Jiang et al. 2005; Kishi et al. 2005; Yoshimura et al. 2005; Barnes et al. 2007; Shelly et al. 2007). Mounting evidence suggests that transcriptional programs control distinct aspects of the development of axons or dendrites, including their growth and branching (Jan and Jan 2003; Goldberg 2004; Polleux et al. 2007). These studies raise the question of whether cell-intrinsic transcriptional mechanisms might also trigger the initial specification of neuronal processes into axons and dendrites, and the establishment of the unique polarized morphology of neurons.

Within the mammalian brain, granule neurons of the developing cerebellum provide a robust system for the study of axon and dendrite development (Ramón y Cajal 1995; Powell et al. 1997). Soon after granule neurons exit mitosis in the external granule layer (EGL) of the developing cerebellum, they begin to extend axons that eventually form the parallel fibers of the cerebellar cortex (Altman and Bayer 1997). Axon growth continues as granule neurons migrate through the molecular and Purkinje cell layers to reach the internal granule layer (IGL). Once granule neurons take up residence in the IGL, 
they elaborate dendrites. Granule neuron axon extension, migration, and dendrite development peak around the second to third week postnatally in the rat cerebellar cortex (Altman and Bayer 1997). Differentiated granule neurons, like other neurons in the brain, are highly polarized, with long axons and much shorter dendrites (Ramón y Cajal 1995).

Studies of neuronal morphogenesis in the cerebellar cortex suggest that distinct transcriptional mechanisms influence specific aspects of the development of axons and dendrites (Stegmüller and Bonni 2005; Kim and Bonni 2007). The transcriptional modulator SnoN is required for parallel fiber axon growth (Stegmüller et al. 2006). Similarly, the development of different phases of granule neuron dendrite development comes under the purview of specific transcription factors. NeuroD promotes the growth and maintenance of dendrites, $\mathrm{Sp} 4$ promotes dendritic pruning, and MEF2A stimulates synaptic dendritic differentiation (Gaudillière et al. 2004; Shalizi et al. 2006; Ramos et al. 2007). These studies suggest that additional undefined transcriptional mechanisms might regulate other aspects of neuronal morphogenesis in the cerebellar cortex, including establishment of the polarized neuronal shape of granule neurons.

Besides granule neurons of the cerebellar cortex, hippocampal pyramidal neurons have been employed in the study of neuronal morphogenesis, especially in axodendritic polarization. Primary hippocampal neurons become polarized in well-defined steps, beginning with the extension of several undifferentiated neurites that express markers of both axons and dendrites, following which the longest process expresses axon-specific markers, and the remaining neurites differentiate into dendrites (Craig and Banker 1994). Studies of neuronal polarization in hippocampal neurons have focused on local events in the neuronal processes (Shi et al. 2003; Schwamborn and Puschel 2004; de Anda et al. 2005; Jiang et al. 2005; Kishi et al. 2005; Yoshimura et al. 2005; Jacobson et al. 2006; Arimura and Kaibuchi 2007). However, a role for cell-intrinsic transcriptional mechanisms in axo-dendritic polarization has not been explored.

The FOXO transcription factors are widely expressed in the developing mammalian brain (Brunet et al. 1999; Hoekman et al. 2006). While biological functions of the FOXO proteins have been characterized outside the nervous system (Burgering and Kops 2002; Tran et al. 2003; Accili and Arden 2004; Arden 2004; Coffer and Burgering 2004; Van Der Heide et al. 2004; Barthel et al. 2005; Carter and Brunet 2007), the function of these factors in unique aspects of neural development have remained to be identified. Interestingly, expression of the FOXO family member FOXO6 is enriched in the brain, including the cerebral cortex and hippocampus, but its function has remained unknown (Jacobs et al. 2003; van der Heide et al. 2005; Hoekman et al. 2006).

In this study, we identify a novel role for the FOXO transcription factors, including the brain-enriched protein FOXO6, in the establishment of neuronal polarity in the mammalian brain. We also identify the polarityassociated protein kinase Pak1 as a critical direct target gene of the FOXO proteins in neuronal polarity. Collectively, our data define the FOXO-Pak1 pathway as a cellintrinsic transcriptional mechanism that establishes neuronal polarity.

\section{Results}

FOXO transcription factors are required for establishment of granule neuron polarity

In situ hybridization analyses have revealed that the transcription factors FOXO1, FOXO3, and FOXO6 are expressed in the mammalian brain at a time when neurons undergo a number of developmental events, including neuronal polarization (Supplemental Fig. 1A,B; Hoekman et al. 2006). In addition, we found by immunoblotting that FOXO1, FOXO3, and FOXO6 are expressed in primary rat cerebellar granule neurons (Supplemental Fig. 1C). The expression of the FOXO proteins increased with maturation in primary granule neurons (Supplemental Fig. 1C). Together, these observations indicate that the FOXO proteins are expressed in developing mammalian brain neurons, and their temporal pattern of expression suggests a possible role in neuronal morphogenesis.

To investigate if the FOXO proteins might contribute to neuronal morphogenesis, we employed a DNA templatebased method of RNAi to express shRNAs targeting the FOXO proteins FOXO1, FOXO3, and FOXO6 (Gaudillière et al. 2002; Lehtinen et al. 2006; Yuan et al. 2008). We confirmed that expression of FOXO shRNAs led to the knockdown of endogenous FOXO1, FOXO3, and FOXO6 in neurons (Fig. 1A). The levels of endogenous FOXO1, FOXO3, and FOXO6 mRNA were reduced within 24-48 h in neurons after transfection with the FOXO RNAi plasmid (Supplemental Fig. 2).

To determine the effect of FOXO knockdown on neuronal morphogenesis, we transfected primary cerebellar granule neurons prepared from postnatal day $6(\mathrm{P} 6)$ rat pups with the FOXO RNAi plasmid (U6/foxo) or control U6 plasmid, together with a GFP expression plasmid to label transfected neurons. FOXO RNAi triggered a striking phenotype in primary granule neurons. A significant proportion of FOXO knockdown neurons displayed a nonpolarized morphology (Fig. 1B,C; Supplemental Fig. 3). The control U6-transfected neurons had a polarized morphology with long Taul-positive, MAP2-negative axons, and short Tau1-negative, MAP2-positive dendrites (Fig. 1D,E). In contrast, the nonpolarized FOXO knockdown granule neurons had multiple morphologically similar processes that were positive for both the axonal marker Taul and the dendrite marker MAP2 (Fig. 1D,E). To quantify the loss of polarization in granule neurons upon FOXO knockdown, we measured the ratio of Taul or MAP2 signal in the longest process compared with the second-longest process, which respectively represent the axon and a dendrite in control neurons (Kishi et al. 2005). Enrichment of Taul was significantly reduced and enrichment of MAP2 signal was significantly increased in the longest process in granule neurons upon FOXO knockdown (Fig. 1F). We subjected control and FOXO 
A
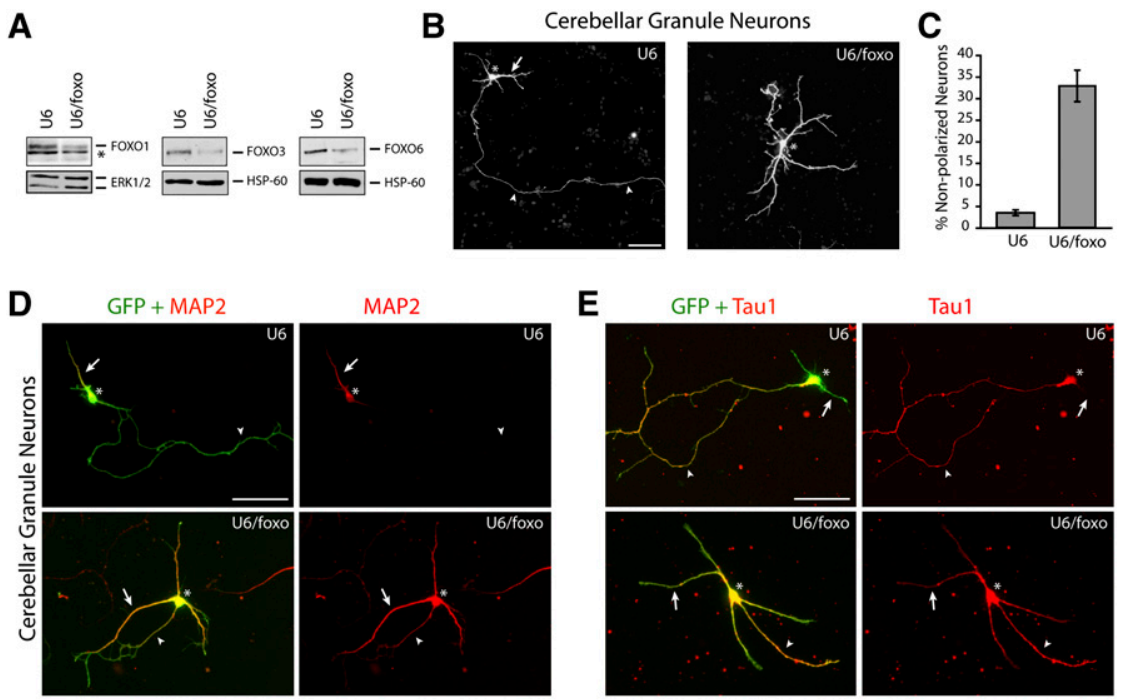

E
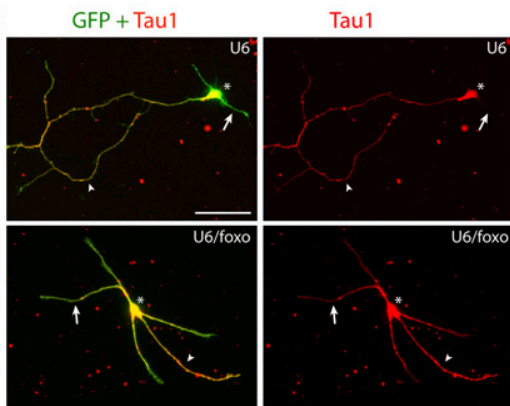

$\mathbf{F}$
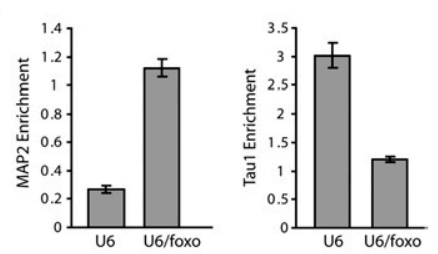

G
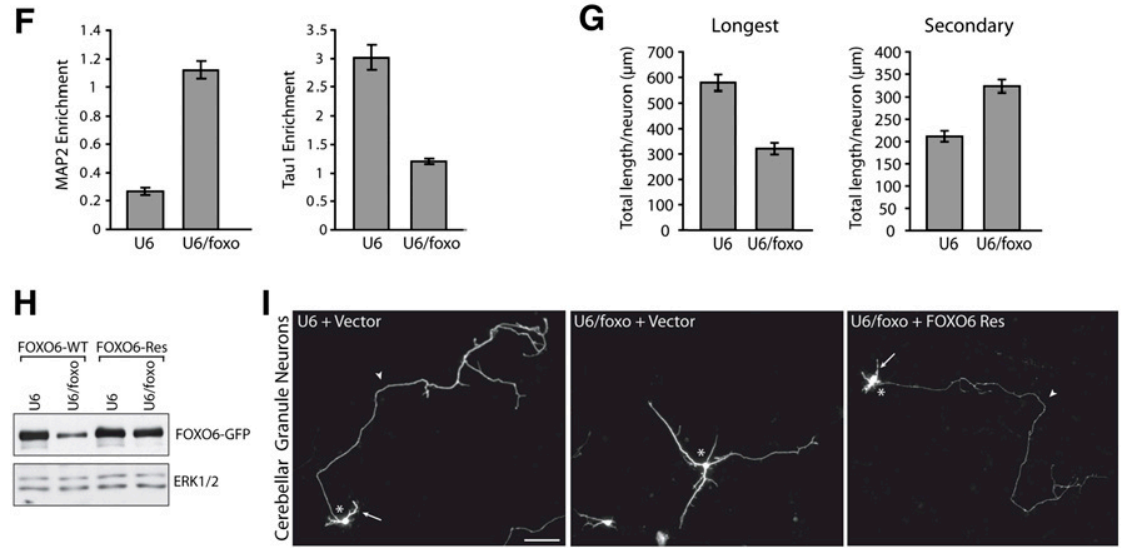

J

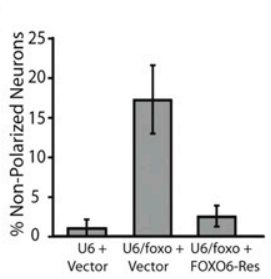

K

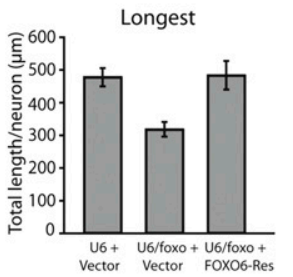

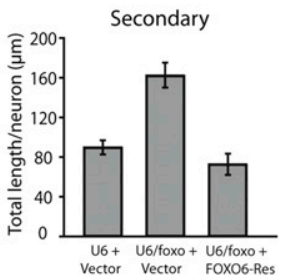

Figure 1. FOXO transcription factors establish neuronal polarity in cerebellar granule neurons. (A) Granule neurons were electroporated before plating using the Amaxa nucleofection kit with the control U6 or U6/foxo RNAi plasmid. Four days after transfection, lysates were subjected to immunoblotting with a FOXO1, FOXO3, or FOXO6 antibody. FOXO RNAi substantially reduced levels of endogenous FOXO1, FOXO3, and FOXO6 in neurons. The asterisk indicates nonspecific band. (B) Cerebellar granule neurons transfected with the control U6 or U6/foxo RNAi plasmid and a GFP expression plasmid were subjected $4 \mathrm{~d}$ after transfection to immunocytochemistry with an antibody to GFP (see Supplemental Fig. 3 for additional lower-magnification panels). Arrows, arrowheads, and asterisks indicate dendrites, axons, and cell body, respectively. Bar, $50 \mu \mathrm{m} .(C)$ Granule neurons transfected and analyzed as in $B$ were scored as polarized or nonpolarized. FOXO knockdown significantly increased the number of neurons that fail to acquire a polarized morphology $(P<$ 0.01 ; $t$-test, $n=3)$. $(D-F)$ Granule neurons were transfected with the Amaxa electroporation device with the control U6 or U6/ foxo RNAi plasmid and the GFP expression plasmid and grown at low density. Five days after transfection, neurons were subjected to immunocytochemistry with the GFP antibody and an antibody to the dendritic marker MAP2 $(D)$ or the axonal marker Taul $(E)$. Enrichment of Tau1 and MAP2 was quantified in F. Tau1 and MAP2 enrichment are defined as the intensity of Taul or MAP2 immunostaining in the longest neurite divided by the intensity in the second-longest neurite. FOXO knockdown neurons displayed significantly increased MAP2 enrichment $(P<0.001 ; t$-test, $n=3)$ and significantly reduced Taul enrichment $(P<$ 0.01 ; $t$-test, $n=3$ ) when compared with control U6-transfected neurons. Arrowheads and arrows point to the longest process and other processes, respectively. Asterisks indicate cell bodies. $(G)$ Morphometric analysis of granule neurons transfected as in $B$ revealed that FOXO RNAi significantly reduced the length of the longest process (axon in control), and concomitantly increased the length of secondary processes (dendrites in control) $(P<$ 0.001 ; $t$-test, 213 neurons measured). (H) Lysates of $293 \mathrm{~T}$ cells transfected with the control U6 or U6/foxo RNAi plasmid together with an expression vector encoding GFP-tagged FOXO6 (FOXO6-WT) or the RNAi-resistant mutant FOXO6 (FOXO6-Res) were subjected to immunoblotting with the GFP antibody (top panel) or an antibody to ERK1/2 (bottom panel). (I-K) Granule neurons transfected with the control U6 or U6/foxo RNAi plasmid, together with the FOXO6-Res expression plasmid or its control vector and an expression plasmid encoding DsRed, were subjected $4 \mathrm{~d}$ after transfection to immunocytochemistry with an antibody to DsRed. FOXO6-Res significantly reduced the percentage of nonpolarized neurons in the background of FOXO RNAi $(P<0.01$; ANOVA, $n=3)$. The length of the longest process (axon in control) was significantly reduced and the length of secondary processes (dendrites in control) was significantly increased upon FOXO RNAi $(P<0.001$; ANOVA, 200 neurons measured), but not in FOXO6-Res-expressing neurons in the background of FOXO knockdown, when compared with control U6-transfected neurons. Arrows, arrowheads, and asterisks indicate dendrites, axons, and cell body, respectively. Bar, $50 \mu \mathrm{m}$.

knockdown granule neurons to morphometric analyses. In control granule neurons, as in most neurons in the brain, the longest process is the axon while the other, shorter processes develop into dendrites. FOXO knockdown neurons exhibited significantly longer secondary processes (dendrites in control), while the longest process (axon in control) was significantly shorter as compared with control U6-transfected neurons (Fig. 1G). In control experiments, FOXO knockdown did not affect the immunoreactivity of markers of post-mitotic granule 
neurons, including the neuron-specific class III $\beta$-tubulin (Tuj1) and the transcription factor MEF2A (Supplemental Fig. 4). In addition, FOXO knockdown did not have adverse effects on cell survival, and thus the impairment of neuronal polarity in FOXO knockdown neurons was not associated with reduced cell survival (Supplemental Fig. 5). Together, these results suggest that knockdown of the FOXO proteins impairs axo-dendritic polarization in granule neurons.

To determine the specificity of the FOXO RNAi-induced neuronal polarity phenotype, we performed a rescue experiment. We generated expression plasmids encoding rescue forms of FOXO1, FOXO3, and FOXO6 by introducing silent mutations in the cDNA encoding the FOXO proteins designed to render them resistant to FOXO RNAi (FOXORes). We confirmed that expression of FOXO shRNAs failed to effectively induce knockdown of FOXO1-Res (Yuan et al. 2008), FOXO3-Res (Lehtinen et al. 2006), and FOXO6-Res (Fig. 1H). We next tested if expression of the rescue forms of FOXO proteins suppresses the FOXO RNAi-induced phenotype in granule neurons. Expression of FOXO1-Res or FOXO3-Res significantly, albeit partially, reversed the FOXO RNAi-induced phenotype in granule neurons (Supplemental Fig. 6A,B). Expression of FOXO6Res restored the polarized morphology of granule neurons in the background of FOXO RNAi (Fig. 1I,J). Expression of the FOXO rescue proteins on their own had little or no effect on polarity in granule neurons (Supplemental Fig. 6C). FOXO6-Res also reversed the dual effect of FOXO RNAi on the growth of the longest and secondary processes in granule neurons (Fig. 1K). Together, these results indicate that the FOXO RNAi-induced phenotype is the result of specific knockdown of FOXO proteins, rather than off-target effects of RNAi or nonspecific activation of the RNAi machinery. Our results also suggest that, among the FOXO proteins, FOXO6 is the prominent though not exclusive member that promotes neuronal polarity.

To further characterize the relative roles of the FOXO proteins in the establishment of neuronal polarity, we generated U6/foxo1, U6/foxo3, and U6/foxo6 RNAi plasmids encoding shRNAs targeting each of the three FOXO proteins specifically (Supplemental Fig. 7A,B). In contrast to FOXO RNAi inducing the knockdown of FOXO1, FOXO3, and FOXO6 (see Fig. 1), knockdown of each of the three FOXO proteins alone failed to impair polarity in granule neurons (Supplemental Fig. 7C), suggesting that FOXO1, FOXO3, and FOXO6 have redundant functions in the establishment of neuronal polarity. Accordingly, the combined expression of FOXO1, FOXO3, and FOXO6 shRNAs impaired polarity in granule neurons, thus phenocopying the effect of FOXO shRNAs (Supplemental Fig. 7D,E). Collectively, our data suggest that FOXO6 collaborates with FOXO1 and FOXO3 to induce neuronal polarity and promote the dual morphogenesis of axons and dendrites.

We next characterized the temporal dynamics of the FOXO RNAi-induced polarity phenotype. In analyses of cohorts of granule neurons, we found that the majority of control P6 neurons acquire a polarized morphology between the first and second day after plating (Fig. 2A).
Remarkably, FOXO RNAi-transfected granule neurons did not convert to a polarized morphology, and remained in a nonpolarized state throughout the course of the analysis.

To determine whether FOXO proteins control the transition from a nonpolarized to a polarized morphology, we performed time-lapse analyses of individual control and FOXO knockdown granule neurons. Neurons were classified into five distinct stages (Powell et al. 1997). Stages 1-2 and stages 3-5 represent nonpolarized and polarized neurons, respectively. At the time of initial observation, both control and FOXO knockdown granule neurons were found in both polarized and nonpolarized morphologies (Fig. 2B,C). During the ensuing $86 \mathrm{~h}$ of observation, control granule neurons that were initially polarized remained polarized, and neurons that were initially nonpolarized converted to a polarized morphology (Fig. 2C). In contrast, FOXO knockdown neurons that were initially nonpolarized did not convert to a polarized morphology throughout the $86 \mathrm{~h}$ of observation (Fig. $2 \mathrm{~B}, \mathrm{C})$. Interestingly, FOXO knockdown neurons that were initially polarized remained polarized (Fig. 2C). Quantification of these analyses revealed that FOXO knockdown blocked polarization in nearly $70 \%$ of neurons that were initially nonpolarized, while only $15 \%$ of control granule neurons that were initially nonpolarized remained nonpolarized at the last time point of observation (Fig. 2D). However, none of the FOXO knockdown or control granule neurons that were initially polarized became nonpolarized at the last time point of observation (Fig. 2C). Together, these results suggest that the FOXO proteins trigger a switch from nonpolarized to polarized morphology in neurons.

\section{FOXO transcription factors orchestrate axo-dendritic polarization in hippocampal neurons}

We next asked if the function of the FOXO transcription factors in the establishment of neuronal polarity is specific to cerebellar granule neurons, or if the FOXO proteins play a generalized role in neuronal polarity in mammalian neurons. We therefore characterized the role of FOXO transcription factors in primary hippocampal neurons, an established system in the study of neuronal polarization (Craig and Banker 1994). Induction of FOXO RNAi in hippocampal neurons significantly increased the number of nonpolarized neurons, leading to a threefold increase in the percentage of nonpolarized neurons as compared with control U6-transfected neurons (Fig. 3A). The large majority of control U6-transfected hippocampal neurons had a polarized morphology. These neurons displayed a long Taul-positive, MAP2-negative axon, and multiple short Tau1-negative, MAP2-positive dendrites (Fig. 3B,C). The nonpolarized FOXO knockdown hippocampal neurons had multiple morphologically similar processes that were both Taul- and MAP2-positive (Fig. 3B,C). Quantification of the ratio of Tau1 or MAP2 signal in the longest process compared with the secondlongest process-which represent the axon and the dendrite in control neurons, respectively (Kishi et al. 
A
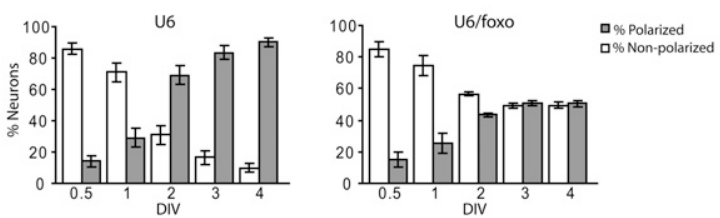

B

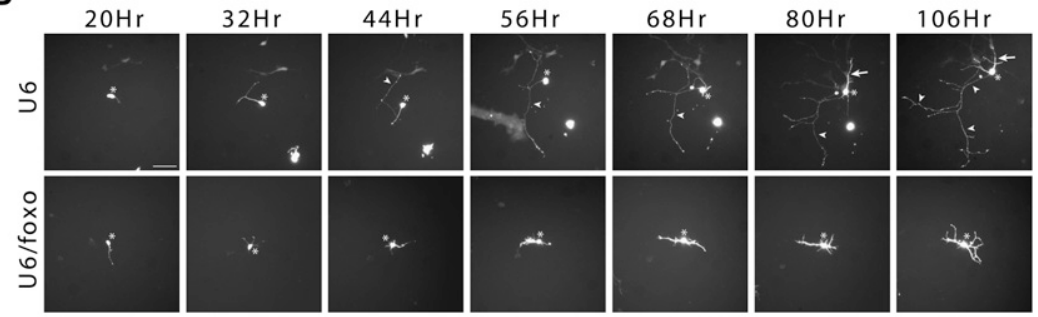

C
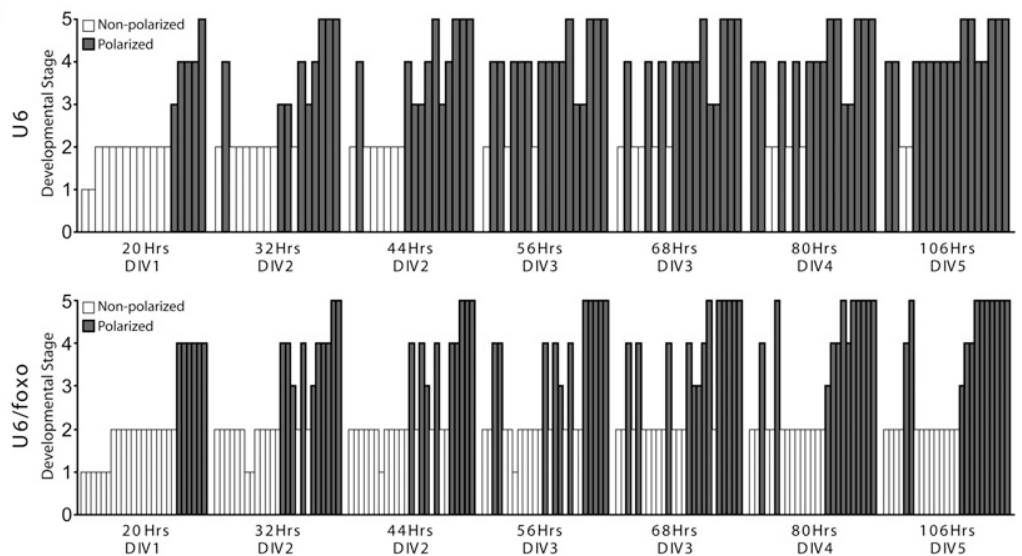

D

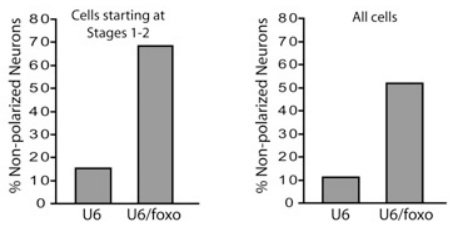

Figure 2. FOXO transcription factors play a critical role in the switch from nonpolarized to polarized morphology in neurons. (A) Granule neurons transfected with the control U6 or U6/foxo RNAi plasmid and the GFP expression plasmid were scored as polarized or nonpolarized at each time point. While a majority of control neurons exhibited a polarized morphology at $2 \mathrm{~d}$ in vitro (DIV2), FOXO RNAi-transfected neurons failed to polarize over time. (B) Granule neurons plated on etched coverslips were transfected $8 \mathrm{~h}$ later with the U6 control or U6/foxo RNAi plasmid together with the GFP expression plasmid. Twenty hours after plating, individual live neurons were imaged in 12 -h intervals over the course of $86 \mathrm{~h}$. Nonpolarized control neurons acquired a polarized morphology within the first $36 \mathrm{~h}$ of observation. In contrast, FOXO knockdown neurons failed to polarize in the same amount of time. Arrows, arrowheads, and asterisks indicate dendrites, axons, and cell body, respectively. Bar, $50 \mu \mathrm{m} .(C, D)$ Quantification of the developmental stage of individual neurons transfected and analyzed as in $B$. Neurons were grouped into five different morphological developmental stages, as described by Powell et al. (1997), with some modification. Stages 1-2 represent nonpolarized neurons bearing no neurites (stage 1), or several unspecified processes (stage 2). Stages 3-5 designate polarized neurons, including bipolar neurons bearing two axon-like processes (stage 3), multipolar neurons with an axon and short dendrites (stage 4), and multipolar complex neurons with long axons and elaborate dendritic arbors (stage 5). The majority of control neurons $(85 \%)$ starting at stages $1-2$ reached the polarized stages $4-5$ by $5 \mathrm{~d}$ in vitro (DIV5), while a large proportion of FOXO knockdown neurons $(68 \%)$ remained in stage 2 . Both control and FOXO knockdown neurons that had already acquired a polarized morphology at the beginning of the analysis remained polarized throughout the course of observation. (DIV) Days in vitro.
2005) - revealed that enrichment of Tau1 and MAP2 signal in the longest process was reduced and increased significantly, respectively, in hippocampal neurons upon FOXO knockdown (Fig. 3D,E). In control experiments, FOXO RNAi did not alter the immunoreactivity of class III $\beta$-tubulin in hippocampal neurons (Supplemental Fig. 8). Together, our results show that, just as in granule neurons, FOXO knockdown impairs the specification of neurites into axons and dendrites in hippocampal neurons.

To establish the specificity of the FOXO RNAi-induced phenotype in hippocampal neurons, we performed rescue experiments. Just as in cerebellar granule neurons, expression of FOXO6-Res in hippocampal neurons reversed the FOXO RNAi-induced phenotype. FOXO6-Res substantially and significantly reduced the number of nonpolarized hippocampal neurons in the background of FOXO knockdown (Fig. 3F,G). Importantly, FOXO6-Res led to a significant increase in Taul enrichment and a concomitant reduction in MAP2 enrichment in the longest process in FOXO knockdown hippocampal neurons (Fig. 3H-J). In control experiments, expression of FOXO6-Res on its own had little or no effect on polarity in hippocampal neurons (data not shown). The rescue experiments suggest that the FOXO RNAi-induced polarization phenotype in hippocampal neurons results from specific FOXO knockdown, rather than off-target effects of the RNAi machinery. Collectively, our findings suggest that FOXO function in neuronal polarization may be generalized in mammalian brain neurons.

\section{FOXO proteins are required in the establishment of neuronal polarity in vivo}

We next determined the role of FOXO proteins in neuronal morphogenesis in vivo. We used an electroporation method of RNAi developed for the postnatal rat cerebellum (Fig. 4A,B; Konishi et al. 2004; Shalizi et al. 2006; Stegmüller et al. 2006). We transfected P3 rat pups 

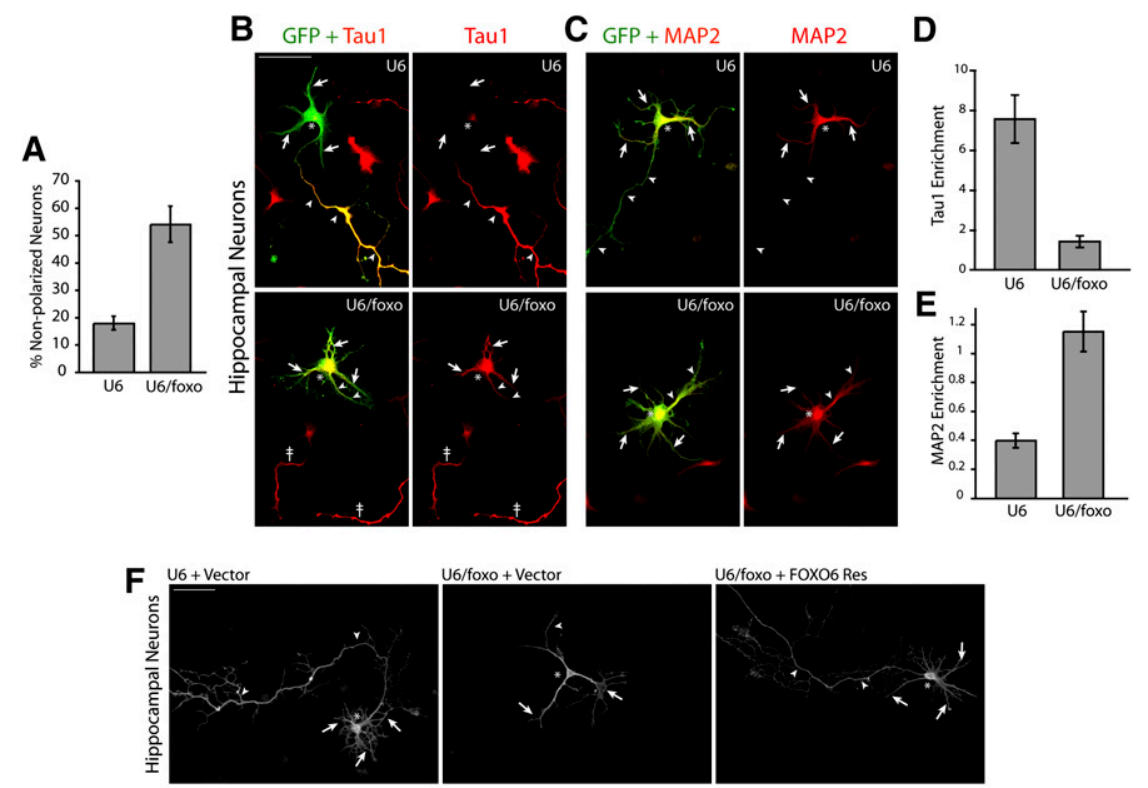

G
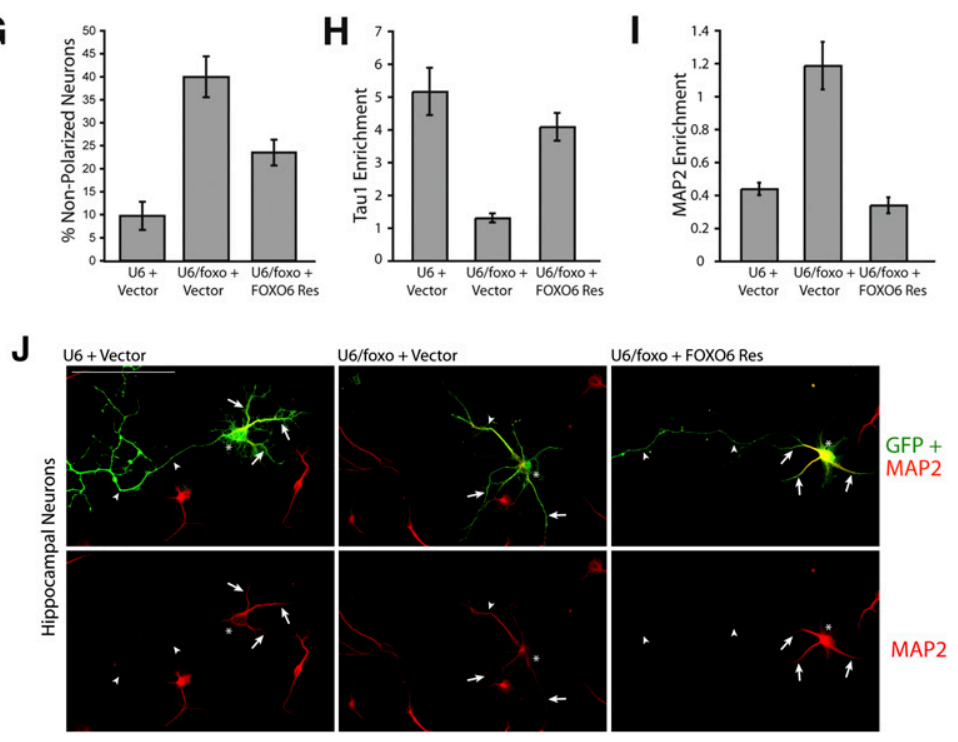

Figure 3. FOXO transcription factors promote axo-dendritic polarization in hippocampal neurons. $(A-E)$ Hippocampal neurons were transfected with the control U6 or U6/foxo RNAi plasmid and the GFP expression plasmid. Four days after transfection, neurons were subjected to immunocytochemistry with the GFP antibody and Taul $(B)$ or MAP2 $(C)$ antibody. The percentage of neurons that failed to acquire a polarized morphology is quantified in $A$. Enrichment of Taul and MAP2 was quantified in $D$ and $E$, respectively. A significant proportion of FOXO knockdown neurons failed to acquire a polarized morphology $(P<0.01$; $t$-test, $n=3)$, and displayed significantly reduced Taul enrichment $(P<0.0001$; $t$-test, 40 neurons measured) and significantly increased MAP2 enrichment $(P<0.0001$; $t$-test, 57 neurons measured) when compared with control U6transfected neurons. Arrowheads and arrows point to longest process and secondary processes, respectively. The asterisks indicate cell bodies. The double dagger points to the Taul-positive axons of untransfected neurons. $(F, G)$ Hippocampal neurons were transfected with the control U6 or U6/foxo RNAi plasmid together with the FOXO6-Res and GFP expression plasmids and were analyzed as in $A$. FOXO6-Res significantly reversed the FOXO RNAi-induced neuronal polarity phenotype $(P<0.05$; ANOVA, $n=3)$. Arrowheads and arrows point to the longest process and other processes, respectively. $(H-J)$ Hippocampal neurons were transfected as in $F$ and, $4 \mathrm{~d}$ later, were analyzed as in $B-E$. Enrichment of Taul and MAP2 was quantified in $H$ and $I$, respectively. A significant proportion of FOXO knockdown neurons failed to acquire a polarized morphology $(P<$ 0.05 ; ANOVA, $n=3$ ), and displayed significantly reduced Tau 1 enrichment $(P<0.0001$; ANOVA, 51 neurons measured) and significantly increased MAP2 enrichment $(P<$ 0.0001; ANOVA, 57 neurons measured) compared with control U6-transfected neurons. These phenotypes were significantly reversed by FOXO6-Res $(P<0.0001$; ANOVA, 51 neurons measured; Taul and $P<0.0001$; ANOVA, 57 neurons measured; MAP2). Representative images of MAP2 immunostaining are shown in $J$.

with a FOXO RNAi plasmid that also encoded GFP (U6/ foxo-cmvGFP) or the control U6-cmvGFP plasmid. Transfected rat pups were returned to moms and examined $5 \mathrm{~d}$ later at P8. Isolated cerebella from P8 animals were subjected to immunohistochemistry using the GFP antibody and an antibody to the Purkinje cell marker calbindin. In control transfected animals, granule neurons residing in the IGL displayed normal dendrite morphology and issued robust granule neuron parallel fiber axons (Fig. 4B,C; Ramón y Cajal 1995; Altman and Bayer 1997).

FOXO knockdown led to a striking phenotype in the cerebellar cortex. IGL granule neurons in FOXO knockdown animals had multiple long secondary processes in the IGL and often lacked clearly defined ascending axons or parallel fibers in the molecular layer (ML) (Fig. 4C;
Supplemental Fig. 9). The remaining parallel fiber axons in FOXO knockdown animals appeared to be less fasciculated than those in control animals, often wandering off the parallel fiber track (data not shown). Quantification of the in vivo phenotype revealed a nearly $50 \%$ increase in the total length of secondary processes in the IGL (dendrites in control) upon FOXO knockdown (Fig. 4D). To quantify the effect of FOXO knockdown on axons, we measured the percentage of IGL granule neurons associated with parallel fibers. More than $80 \%$ of the IGL granule neurons in control U6-transfected animals were associated with parallel fibers. In contrast, only $48 \%$ of the IGL granule neurons in FOXO knockdown animals were associated with parallel fibers (Fig. 4E). To determine the specificity of the FOXO RNAi-induced neuronal phenotype 
A

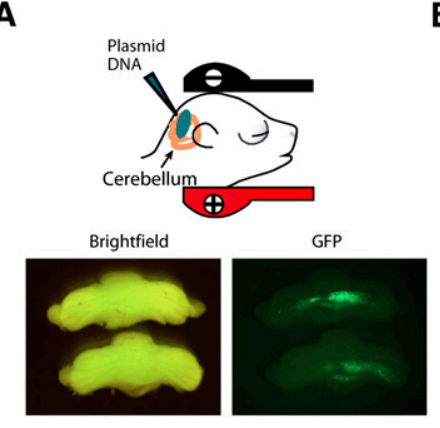

B GFP

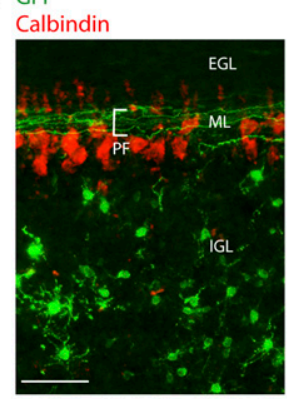

C
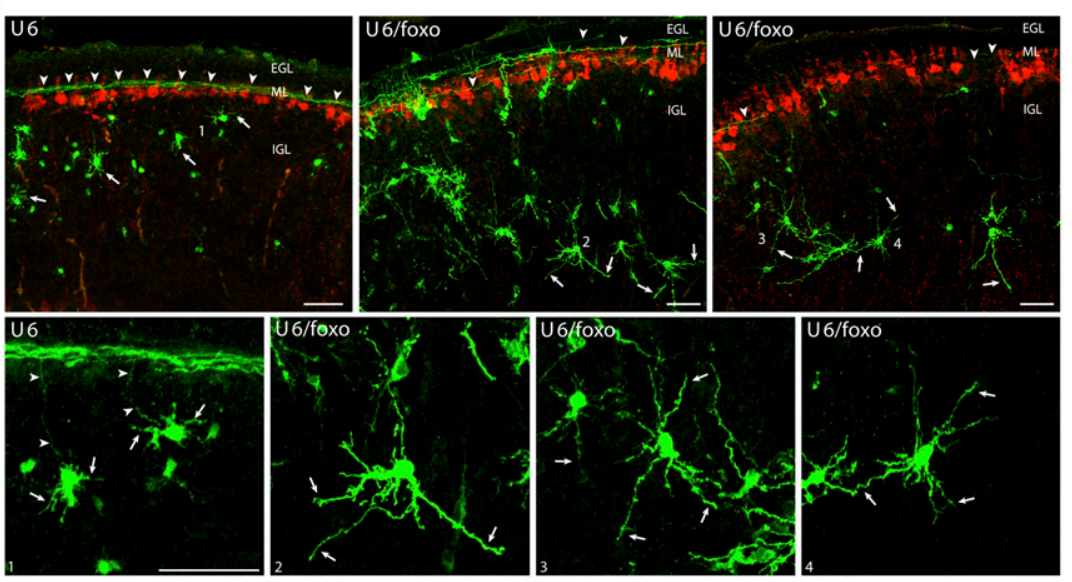

D

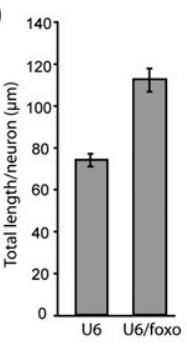

E

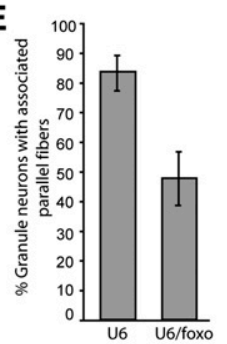

F
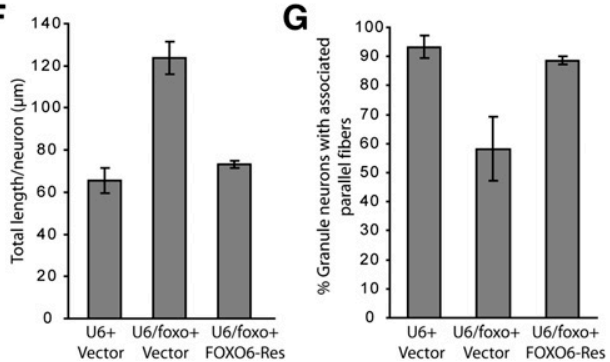

Figure 4. FOXO knockdown disrupts the establishment of neuronal polarity in the cerebellar cortex in vivo. $(A, B) \mathrm{P} 3$ rat pups were injected in the cerebellum with a GFP expression plasmid and then subjected to electroporation. Five days later, at P8, pups were sacrificed and coronal sections of cerebella were subjected to immunohistochemistry with a monoclonal antibody to GFP (green) and a rabbit polyclonal antibody to calbindin (red), the latter to label Purkinje cells. Transfected GFP-positive cerebellar granule neurons bear dendrites and have associated parallel fibers (PF) along the ML. Bars, $50 \mu \mathrm{m} .(C)$ Coronal sections of cerebella electroporated as in $A$ with the control U6-cmvGFP or U6/foxo-cmvGFP RNAi plasmid were subjected to immunohistochemistry with the GFP antibody (green) and the calbindin antibody (red). The bottom panels show a higher magnification of the numbered cells. In control animals (U6), granule neurons in the IGL were typically associated with parallel fibers. In contrast, FOXO knockdown (U6/foxo) led to loss of associated parallel fibers. Concomitant with the decrease in parallel fiber abundance, the length of secondary processes in the IGL was increased in granule neurons in FOXO knockdown animals as compared with granule neurons in control transfected animals. Arrows and arrowheads indicate secondary processes in the IGL (dendrites in control animals) and parallel fibers, respectively. $(D)$ Quantification of total length of secondary processes in the IGL of granule neurons in animals electroporated and analyzed as in $C$. FOXO knockdown significantly increased the length of secondary processes in the IGL in granule neurons $(P<0.001 ; t$-test, 335 neurons measured). (E) Quantification of parallel fiber phenotype upon FOXO knockdown in vivo. The percentage of granule

neuron somas in the IGL that were associated with parallel fibers was significantly reduced in FOXO knockdown animals as compared with control transfected animals $(P<0.001 ; t$-test, $n=3,811$ neurons measured). $(F, G)$ P8 rat pups electroporated at P3 with the control U6-cmvGFP or U6/foxo-cmvGFP RNAi plasmid together with the FOXO6-Res expression plasmid or its control vector were analyzed as in A-E. Expression of FOXO6-Res in the background of FOXO knockdown in vivo significantly reduced the length of secondary processes in the IGL (dendrites in control animals) $(P<0.01$; ANOVA, $n=3,216$ neurons measured) and significantly increased the number of parallel fibers associated with IGL granule neurons $(P<0.05$; ANOVA, $n=3,2655$ neurons measured $)$ as compared with FOXO knockdown animals.

in the cerebellar cortex, we performed a rescue experiment in vivo. We found that expression of FOXO6-Res reversed the effect of FOXO RNAi on parallel fibers and length of secondary processes in the IGL (dendrites in control) (Fig. $4 \mathrm{~F}, \mathrm{G})$. Thus, just as in primary neurons, the FOXO RNAiinduced polarity phenotype in vivo is the result of specific FOXO knockdown. Collectively, these findings support the conclusion that FOXO proteins play a key role in the establishment of neuronal polarity in vivo.

We also examined the effect of FOXO knockdown on the polarized morphology of granule neurons in vivo at a later stage of brain development. We found that the FOXO knockdown-induced phenotype was sustained in rat pups at P12, $9 \mathrm{~d}$ after electroporation. In particular, granule neurons in FOXO knockdown animals had fewer associated parallel fibers than in control U6-transfected animals (Fig. 5A,E). We also observed a substantial increase in the total length of secondary processes in the IGL in FOXO knockdown animals as compared with control transfected animals at P12 (Fig. 5A,B,D). In addition, while a substantial fraction of dendrites in control transfected animals at P12 harbored dendritic claws at their ends, representing sites of post-synaptic differentiation (Shalizi et al. 2006), the long and aberrant secondary processes in the IGL in FOXO knockdown animals had a substantially lower number of dendritic 

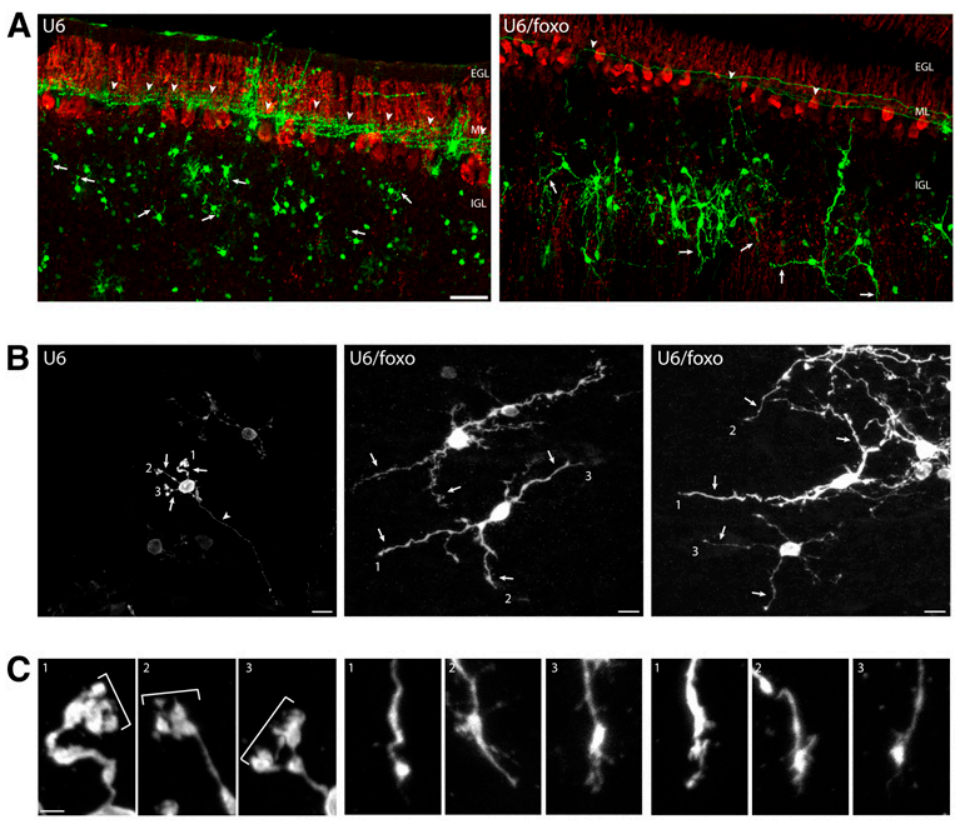

D
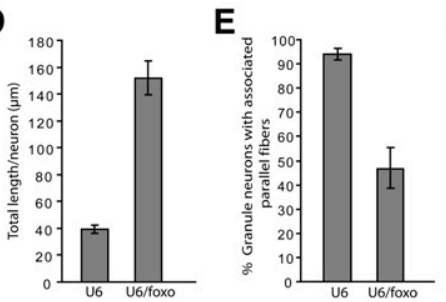

$\mathbf{F}$

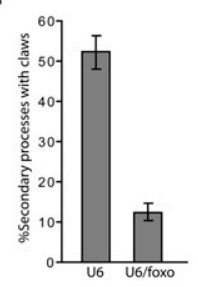

Figure 5. FOXO knockdown-induced impaired neuronal polarity phenotype in vivo is sustained in later stages of development. (A) P3 rat pups were injected in the cerebellum with the control U6-cmvGFP or U6/foxocmvGFP RNAi plasmid and then subjected to electroporation. Nine days, later at P12, pups were sacrificed and coronal sections of cerebella were subjected to immunohistochemistry with the GFP (green) and calbindin (red) antibodies, the latter to label Purkinje cells. In control animals (U6), granule neurons in the IGL were typically associated with parallel fibers. The FOXO knockdowninduced loss of parallel fibers was sustained at this later stage of development (P12). In addition, secondary processes in the IGL (dendrites in control) appeared to be much longer in FOXO knockdown animals as compared with control animals. Arrows and arrowheads indicate dendrites and parallel fibers, respectively. Bar, $50 \mu \mathrm{m} .(B, C)$ Higher magnification of granule neurons in the cerebellar cortex in animals electroporated and analyzed as in $A$. The numbered dendritic tips shown in $B$ are magnified in $C$. Mature dendrites in control animals bear dendritic claws at their ends (indicated by brackets), which represent characteristic post-synaptic structures (Shalizi et al. 2006). In contrast, the aberrant long secondary processes in the IGL in FOXO knockdown animals have tapered ends lacking dendritic claws. Bars: $B, 50 \mu \mathrm{m} ; C, 10 \mu \mathrm{m} .(D)$ Quantification of total length of secondary processes in the IGL of granule neurons in animals electroporated and analyzed as in A. FOXO knockdown significantly increased total secondary process length in granule neurons $(P<0.001$; $t$-test, $n=3$ brains, 172 neurons measured). $(E)$ Quantification of parallel fiber phenotype upon FOXO knockdown in vivo. The percentage of granule neurons in the IGL that were associated with parallel fibers was significantly reduced in FOXO knockdown animals as compared with control transfected animals $(P<0.01 ; t$-test, $n=3$ brains, 809 neurons measured). (F) Quantification of the number of dendritic claws in control and FOXO knockdown animals. FOXO knockdown significantly reduced the number of secondary processes in the IGL (dendrites in control) bearing claws $(P<0.005 ; t$-test, $n=3,141$ neurons measured).

claws (Fig. 5B,C,F). These results suggest that the FOXO knockdown-induced impairment in neuronal polarity is sustained in the developing cerebellar cortex in vivo.

\section{Identification of Pak1 as a downstream mediator of FOXO-dependent neuronal polarity}

We next investigated the mechanism by which the FOXO transcription factors promote neuronal polarity. We measured the effect of FOXO knockdown on an array of genes encoding proteins that directly control neuronal polarization. The polarity transcriptome selected in these analyses included genes encoding the PAR polarity complex proteins, protein kinases, GTPases, GEFs, signaling proteins, kinesin family motor proteins, and scaffold proteins. Using real-time RT-PCR analysis, we measured the abundance of mRNA encoded by each of these genes in control and FOXO knockdown granule neurons. We found that FOXO knockdown reduced the expression of several polarity genes-including Par6, Pak1, R-Ras, APC, and $C R M P 2$ - suggesting that FOXO proteins may control a program of gene expression dedicated to neuronal polarity (Fig. 6A). Among these genes, Pak1 was the most robustly down-regulated gene (Fig. 6A). These results suggest that
Pak1 might represent a target of the FOXO transcription factors in granule neurons.

We first assessed whether Pak1 might be regulated in granule neurons during the process of polarization. In time-course analyses, we found that Pak1 mRNA abundance increases in granule neurons, preceding the onset of polarization (Figs. 2A, 6B). Consistent with these in vitro results, Pak1 mRNA levels also increased during the period of granule neuron polarization in the developing rat cerebellum (Fig. 6C). Concomitant with the increase in mRNA levels, Pak1 protein expression also increased during polarization in primary granule neurons and in the cerebellum (Fig. 6D,E). The increase in Pak1 mRNA and protein levels correlated tightly with the expression profile of the FOXO proteins in granule neurons (see Supplemental Fig. $1 \mathrm{C}$ ), suggesting that the FOXO proteins might regulate Pak1 expression in developing neurons. Consistent with this conclusion, we confirmed that FOXO knockdown led to the down-regulation of Pak1 protein in primary granule neurons (Fig. 6F). Taken together, our results suggest that Pak1 is transcriptionally up-regulated during neuronal polarization, and that Pak1 is a target gene of the FOXO transcription factors in neurons. 
A

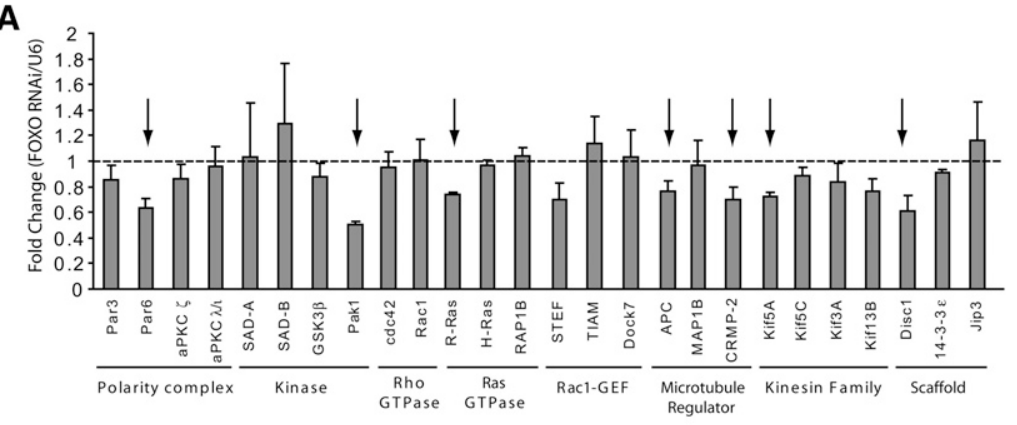

B

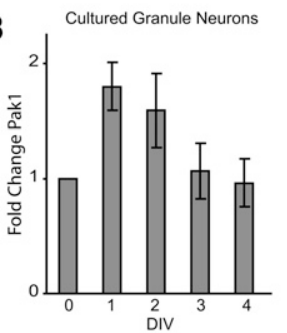

G

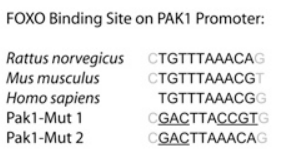

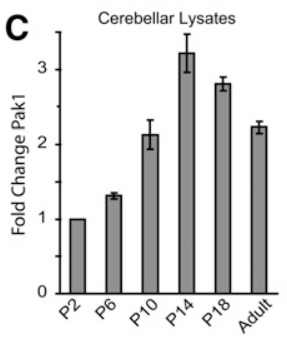

H

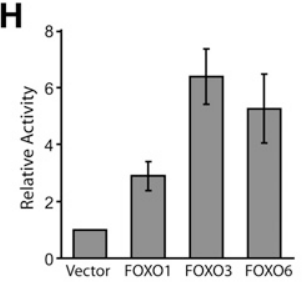

D

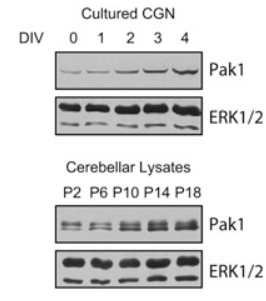

I

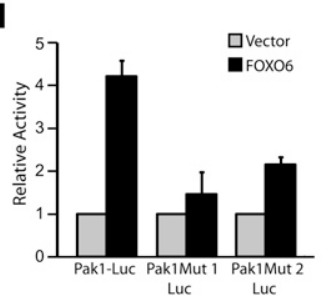

F
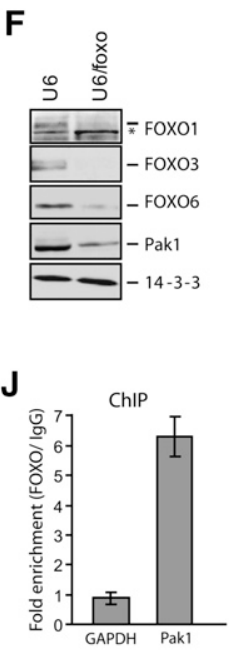

Figure 6. Identification of Pak1 as a direct target of FOXO transcription factors in neurons. $(A)$ Granule neurons were transfected at high efficiency with the control U6 or U6/foxo RNAi plasmid. Two days later, RNA was extracted and reverse-transcribed for use in quantitative PCR of genes encoding proteins implicated in the establishment of neuronal polarity. Knockdown of FOXO transcription factors significantly reduced expression of several polarity genes. Pak1 expression was the most robustly down-regulated of all of the genes tested. Arrows indicate genes that are significantly reduced in FOXO knockdown neurons as compared with U6 control transfected neurons $(P<0.05$; $t$-test, $n=3) .(B, C)$ Pak1 mRNA abundance was assessed by quantitative RT-PCR in cultured granule neurons $(B)$ or in the cerebellum $(C)$ at the indicated time points. Pak1 mRNA abundance increases preceding the onset of polarization. $(D, E)$ Pak1 protein expression was analyzed by immunoblotting of lysates prepared from cultured granule neurons $(D)$ or from cerebellar lysates $(E)$ at the indicated time points. Pak1 expression increases during the period of polarization. $(F)$ Granule neurons were transfected at high efficiency with the control U6 or the U6/foxo plasmid. Four days later, lysates were prepared and subjected to immunoblotting with the indicated antibodies. FOXO knockdown triggered the down-regulation of Pak1 protein levels in neurons. $(G)$ The Pak1 promoter contains putative FOXO-binding sites. Sequence alignment of a fragment of rat, mouse, and human Pak1 promoters is shown along with the engineered mutations in the putative FOXO-binding sites. $(H)$ Granule neurons were transfected with a luciferase reporter gene under the control of a 1.4-kb region of the rat Pak1 promoter containing conserved FOXO-binding sites (Pak1Luc) and an expression plasmid encoding FOXO1, FOXO3, FOXO6, or the control plasmid, together with a Renilla reporter to serve as control for transfection efficiency. Expression of FOXO transcription factors significantly increased the activity of the Pak1-Luc reporter gene $(P<0.01$; ANOVA, $n=3)$. (I) Granule neurons were transfected with a plasmid encoding FOXO6 or its control vector together with Pak1-Luc or the Pak1 promoter containing mutations within the putative FOXO-binding site (Pak1 Mut 1/2-Luc) and the tk-Renilla reporter. Expression of FOXO6 robustly induced the expression of the Pak1-Luc reporter gene $(P<0.001$; ANOVA, $n=3)$, but failed to effectively induce the expression of the Pak1 Mut 1-Luc or the Pak1 Mut 2-Luc reporter gene. $(J$ ) FOXOs occupy the promoter of the endogenous Pak1 gene in granule neurons by ChIP analysis. Granule neuron chromatin was subjected to immunoprecipitation with a control IgG antibody or with antibodies to FOXO1, FOXO3, and FOXO6. Immunoprecipitates were analyzed by quantitative PCR using primers designed to amplify the promoter of the Pak1 gene encompassing the putative FOXO-binding sequence or the first exon of the GAPDH gene as control. Data are plotted as the relative FOXO/IgG immunoprecipitation efficiency. FOXO occupancy at the Pak1 gene is significant relative to the GAPDH gene $(P<0.005$; $t$-test, $n=3)$.

We next determined if the FOXO proteins directly regulate Pak1 gene expression in neurons. Interrogation of the Pak1 gene promoter revealed two contiguous putative FOXO-binding sites conserved in humans, mice, and rats, located at 1223 nucleotides (nt) upstream of the
Pak1 transcriptional start site (Fig. 6G). Expression of FOXO1, FOXO3, or FOXO6 stimulated the expression of a luciferase reporter gene controlled by Pakl promoter sequences that included the putative FOXO-binding sequence (Pak1-luciferase) (Fig. 6H). Importantly, although 
FOXO6 induced the expression of the wild-type Pak1luciferase reporter gene, FOXO6 failed to induce the expression of Pak1-luciferase reporter genes in which the FOXO-binding sequences were mutated (Fig. 6I). In chromatin immunoprecipitation (ChIP) analyses, we found that endogenous FOXO proteins occupy the endogenous Pak1 gene promoter in granule neurons (Fig. 6J). Taken together, these results suggest that the FOXO proteins directly stimulate Pak1 transcription in neurons.

The protein kinase Pak1 has been demonstrated recently to play a critical role in the establishment of neuronal polarity in both primary neurons and the rodent brain in vivo (Jacobs et al. 2007; Causeret et al. 2009). We asked whether Pak1 might mediate the ability of the FOXO transcription proteins to promote neuronal polarity. We first tested the effect of inhibition of endogenous Pak1 in neurons using an RNAi plasmid encoding shRNAs that induce specific knockdown of Pak1 (Jacobs et al. 2007). Pak1 RNAi induced the knockdown of endogenous Pak1 to levels comparable with the downregulation of Pak1 upon FOXO knockdown (Supplemental Fig. 10). Pak1 knockdown strongly increased the number of nonpolarized neurons (Fig. 7A; Supplemental Fig. 11). Quantification of the percentage of nonpolarized neurons revealed that Pak1 knockdown phenocopied the effect of FOXO knockdown on neuronal polarity (Fig. 7A). In other experiments, we found that, although FOXO knockdown and Pak1 knockdown each significantly increased the number of nonpolarized neurons, the combination of FOXO and Pak1 knockdown did not additively increase the number of nonpolarized neurons (Fig. 7B). Together, these results are consistent with the conclusion that FOXO and Pak1 operate in a common pathway to control neuronal polarization.

We next determined the effect of expression of exogenous Pak1 on the ability of FOXO RNAi to impair neuronal polarity. If the down-regulation of Pak1 upon FOXO knockdown is critical to the phenotype of impaired neuronal polarity, exogenous expression of Pak1 would be predicted to reverse the FOXO phenotype. In agreement with this prediction, we found that the expression of Pak1 substantially restored the establishment of neuronal polarity in FOXO knockdown neurons (Fig. 7C,D). Pak1 expression also restored the length of the longest process to control levels in FOXO knockdown neurons (Fig. 7E). In addition, Pak1 expression reduced the ability of FOXO knockdown to stimulate growth of secondary processes (Fig. 7E). Thus, the expression of Pak1 overrides the effect of FOXO knockdown on neuronal polarity in primary granule neurons.

In another set of experiments, we assessed the role of Pak1 in the ability of the FOXO proteins to promote the establishment of neuronal polarity in the rodent brain in vivo. We asked if expression of Pak1 might suppress the FOXO knockdown-induced phenotype of impaired neuronal polarity. Strikingly, we found that expression of exogenous Pak1 in the cerebellar cortex in rat pups dramatically reversed the FOXO knockdown-induced impaired neuronal polarity phenotype in vivo (Fig. 7F$\mathrm{H})$, suggesting that Pak1 mediates the ability of the FOXO proteins to promote neuronal polarity in the mammalian brain. Collectively, our findings suggest that Pak1 is a critical direct target of the FOXO transcription factors in the establishment of neuronal polarity.

\section{Discussion}

In this study, we discovered a novel function for the FOXO proteins as key regulators of neuronal polarity in the mammalian brain. The FOXO transcription factors, including the brain-enriched family member FOXO6, play an essential role in the specification of undifferentiated neurites in post-mitotic neurons into axons and dendrites. We also elucidated a mechanism that underlies FOXOdependent neuronal polarization. The FOXO transcription factors induce the expression of the protein kinase Pak1, which in turn mediates FOXO function in the establishment of neuronal polarity. Our findings suggest that the machinery that locally establishes polarity in neuronal processes is tightly regulated by a FOXO-dependent transcriptional mechanism in the nucleus.

Although FOXO proteins have been implicated in the control of cell survival in mammalian neurons as in other systems (Brunet et al. 1999; Lehtinen et al. 2006; Yuan et al. 2008), our study uncovers a novel function for these transcription factors in a unique aspect of neural development: the establishment of neuronal polarity. Among the FOXO transcription factors, expression of FOXO6 is enriched in the brain, but its function remained unknown (Jacobs et al. 2003; van der Heide et al. 2005). We found that FOXO6, in collaboration with the other FOXO proteins, plays an important role in neuronal polarity. Transcriptional regulators have been implicated in driving distinct aspects of the morphogenesis of axons or dendrites at developmental phases that follow polarization, from growth and branching to post-synaptic differentiation of dendrites (Grueber et al. 2003; Aizawa et al. 2004; Gaudillière et al. 2004; Hand et al. 2005; Shalizi et al. 2006; Stegmüller et al. 2006; Ramos et al. 2007). The identification of an essential role for the FOXO proteins in the establishment of neuronal polarity bolsters the concept that different transcription factors are dedicated to distinct phases of neuronal morphogenesis in the mammalian brain.

Studies of neuronal polarization have focused on characterization of mechanisms that act locally within neuronal processes (Craig and Banker 1994; Jan and Jan 2003; Shi et al. 2003; Schwamborn and Puschel 2004; Zhou et al. 2004; de Anda et al. 2005; Jiang et al. 2005; Kishi et al. 2005; Yoshimura et al. 2005; Barnes et al. 2007; Shelly et al. 2007). In addition to the Par3/Par6/aPKC protein complex, several other proteins, including the GTPase Cdc42 and the protein kinase Pak1, form components of a growth cone machinery that promotes axonal specification of undifferentiated neuronal processes (Jacobs et al. 2007). In this study, we identified a requirement for a FOXO-dependent transcriptional mechanism in the establishment of neuronal polarity. By controlling the expression of components of the polarity machinery, the FOXO proteins may establish the competence of neuronal 

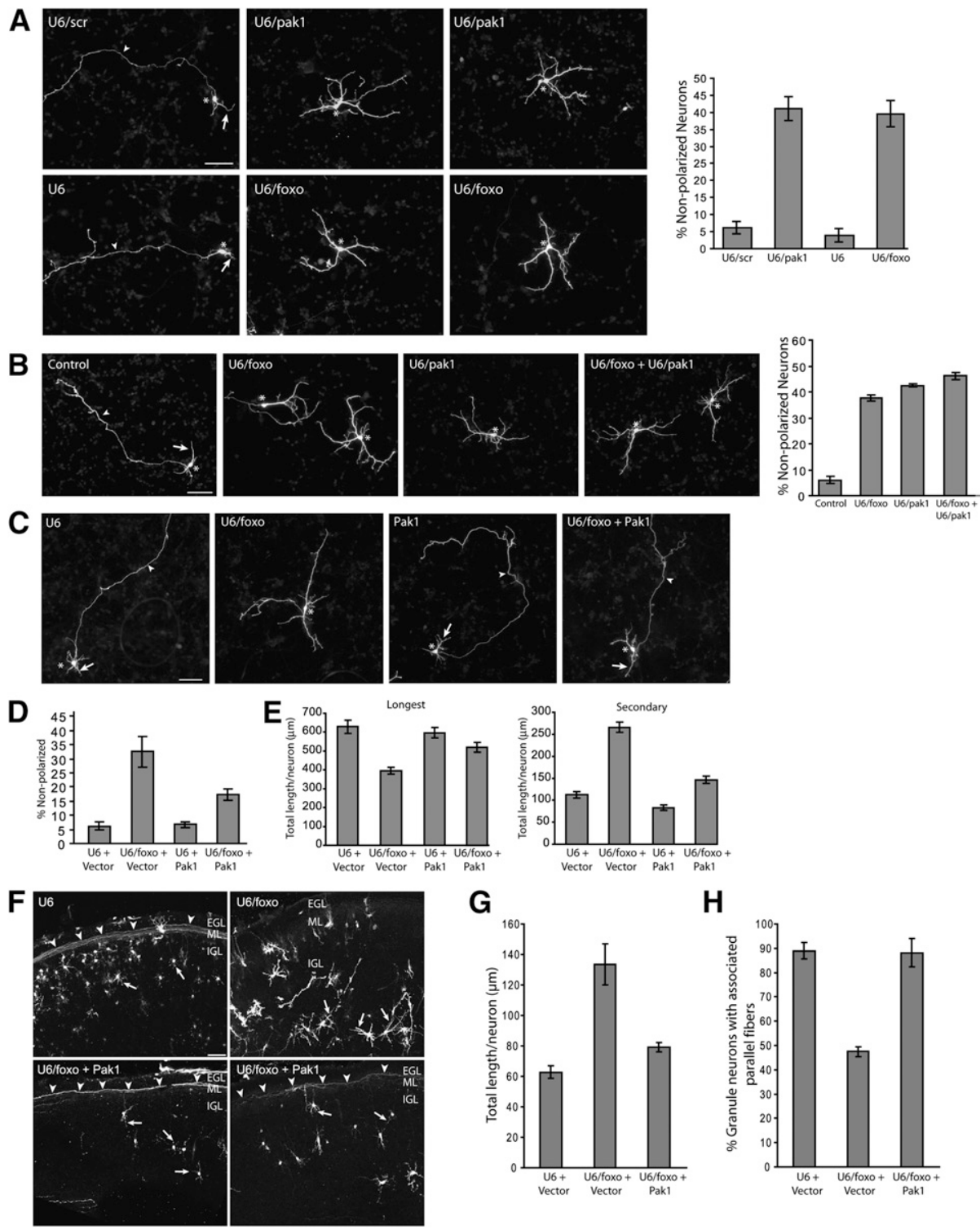

Figure 7. The polarity-associated protein kinase Pak1 mediates FOXO-dependent neuronal polarity. $(A)$ Granule neurons transfected with the control U6, U6/foxo, U6/scr, or U6/pak1 RNAi plasmid and the GFP expression plasmid were subjected $4 \mathrm{~d}$ after transfection to immunocytochemistry with the GFP antibody. Knockdown of Pak1 significantly increased the number of nonpolarized neurons as compared with control U6/scr $(P<0.0001$; ANOVA, $n=3)$ and phenocopied FOXO knockdown. Bar, $50 \mu \mathrm{m}$. $(B)$ Granule neurons were transfected with the control U6, U6/foxo, or U6/pak1, or both the U6/foxo and U6/pak1 RNAi plasmids, together with the GFP expression plasmid and subjected to immunocytochemistry $4 \mathrm{~d}$ later. While individual Pak1 or FOXO knockdown increased the number of nonpolarized neurons $(P<0.0001$; ANOVA, $n=3)$, simultaneous FOXO and Pak1 knockdown did not additively increase the number of nonpolarized neurons as compared with Pak1 knockdown. $(C)$ Granule neurons transfected with the control U6 or U6/foxo RNAi plasmid together with a plasmid expressing Pak1 or its control vector and the GFP expression plasmid were subjected $4 \mathrm{~d}$ after transfection to immunocytochemistry with the GFP antibody. $(D)$ Expression of Pak1 significantly reduced the percentage of nonpolarized neurons in the background of FOXO RNAi $(P<0.01$; ANOVA, $n=3)$. $(E)$ Morphometric analysis shows that the length of the longest process (axon in control) was significantly reduced and the length of secondary processes (dendrites in control) was significantly increased upon FOXO RNAi $(P<0.0001$; ANOVA, $n=3)$. Pak1 expression in the background of FOXO RNAi significantly increased the length of the longest process and significantly reduced the length of secondary processes as compared with FOXO RNAi alone $(P<0.001 ;$ ANOVA, $n=3)$. A total of 636 neurons were measured. $(F-H)$ Coronal sections of P8 rat pups electroporated at P3 with the control U6-cmvGFP or U6/foxocmvGFP RNAi plasmid together with the Pak1 expression plasmid or its control vector were subjected to immunohistochemistry with the GFP antibody. Expression of Pak1 in the background of FOXO knockdown in vivo significantly reduced the length of secondary processes in the IGL $(P<0.05$; ANOVA, $n=3)$ and significantly increased the number of parallel fibers associated with IGL granule neurons $(P<0.01$; ANOVA, $n=3)$ as compared with FOXO knockdown animals. Arrows and arrowheads indicate dendrites and parallel fibers, respectively. Bar, $50 \mu \mathrm{m}$. 
processes to undergo axo-dendritic specification. Thus, the FOXO proteins may control the timing of neuronal polarization during brain development. It will be interesting to determine if transcriptional control of neuronal polarization by the FOXO proteins may also allow the coordination of this fundamental process with other major developmental events, including neuronal migration and survival.

Identification of Pak1 as a direct target of FOXO proteins provides compelling evidence for a link between FOXO-dependent transcription in the nucleus and the expression of a protein that acts in the periphery of the neuron to promote neuronal polarization. Although posttranslational regulation of Pak1 has been characterized (Bokoch 2003; Arias-Romero and Chernoff 2008), our study presents the first evidence of transcriptional regulation of this important polarity regulator. Pak1 appears to promote axonal specification by controlling both the actin and microtubule cytoskeleton in neuronal processes. Pak1 activation of a LIM kinase (LIMK)-cofilin pathway stimulates F-actin assembly (Edwards et al. 1999|, while Pak1-induced phosphorylation and consequent inhibition of the microtubule-severing protein Stathmin/ Op18 promotes microtubule assembly (Wittmann et al. 2004). The link with Pak1 may thus allow the FOXO proteins to control both actin and microtubule dynamics in polarizing neurons.

An important question for future studies is the role of other putative downstream targets of the FOXO transcription proteins in the control of neuronal polarity. Besides Pak1, a number of polarity genes-including Par6, $R$-Ras, APC, and CRMP2-were down-regulated in FOXO knockdown neurons. Expression of exogenous Par6 or Disc1 failed to reverse the FOXO RNAi-induced impaired polarity phenotype in granule neurons (data not shown), highlighting thus far the importance of Pak1 in mediating the ability of FOXO proteins to promote neuronal polarity. The functions of different components of the local polarity machinery are intimately linked. Accordingly, Pak1 is activated by Cdc42, which in turn is an effector of the Par3/Par6/aPKC complex, suggesting that Pak1 operates downstream from the Par polarity complex. Therefore, regulation of $P a k 1$ gene expression on its own may allow the FOXO proteins to indirectly but critically influence the function of the Par polarity complex.

Identification of the link between the FOXO proteins and Pak1 also points to novel functions for these proteins beyond neuronal polarity in diverse biological settings. As a novel direct target gene of the FOXO proteins, Pak1 may contribute to the panoply of cellular processes regulated by FOXO proteins, including differentiation, metabolism, and oxidative stress responses (Burgering and Kops 2002; Tran et al. 2003; Accili and Arden 2004; Coffer and Burgering 2004; Van Der Heide et al. 2004; Barthel et al. 2005). Conversely, our findings suggest novel functions for the FOXO transcription factors based on their requirement for Pak1 gene expression. Pak1 promotes multiple aspects of neuronal development in addition to polarity, including dendritic spine morphogenesis and synapse differentiation (Hayashi et al. 2004,
2007; Nikolic 2008). The expression of Pak1 and FOXO proteins continued to increase in neurons after the onset of polarization (Fig. 6D,E; Supplemental Fig. 1). Therefore, the FOXO-Pak1 pathway may also play a role in post-synaptic dendritic differentiation, with potential implications beyond development, including synaptic plasticity in the adult brain.

Outside the nervous system, Pak1 is thought to contribute to mitotic progression in cycling cells, a function that may also be relevant to neuronal progenitor proliferation in the developing brain (Kumar et al. 2006; Nikolic 2008). The FOXO proteins are activated at the G2/M phase in cycling cells (Alvarez et al. 2001; Yuan et al. 2008). Collectively, these observations suggest the possibility that a FOXO-Pak1 transcriptional pathway may also contribute to mitotic progression in dividing cells. This raises intriguing parallels between mitotic pathways in cycling cells and regulation of polarity in post-mitotic neurons.

Beyond normal development and homeostasis, Pak1 upregulation in the absence of gene amplification has been observed in malignant tumors, including brain tumors, but the mechanisms of Pak1 gene regulation in tumorigenesis have remained unexplored (Kumar et al. 2006). Our findings raise the interesting possibility that FOXO transcription may contribute to Pak1 up-regulation in tumors.

The identification of the FOXO signaling pathway in the establishment of neuronal polarity in the developing brain also raises the prospect for a better understanding of the control of axonal responses in the mature nervous system. In particular, characterization of the FOXO transcription factors as cell-intrinsic promoters of neuronal polarization may provide clues as to how neurons in the adult mammalian CNS lose the capacity to extend axons following injury and disease.

\section{Materials and methods}

\section{Transfection and immunocytochemistry}

Primary cerebellar granule neurons were prepared from P6 LongEvans rat pups as described (Konishi et al. 2002). One day after culture preparation, neurons were treated with cytosine arabinofuranoside (AraC) at a final concentration of $10 \mu \mathrm{M}$ to prevent glial proliferation. For morphology assays, granule neurons were transfected $8 \mathrm{~h}$ after plating using a modified calcium phosphate method (Konishi et al. 2002, 2004; Gaudillière et al. 2004). High-efficiency transfection of granule neurons for biochemical analyses was achieved using a nucleofection method with the Amaxa electroporation device. To visualize endogenous MAP2 and Tau 1, granule neurons were transfected with the Amaxa electroporation device and plated at a density of 100-200 cells per square millimeter. For time-lapse analyses, neurons were plated on etched coverslips (Bellco) and transfected $8 \mathrm{~h}$ later. To rule out the possibility that the effects of RNAi or protein expression on neuronal morphogenesis were due to any effects of these manipulations on cell survival, the anti-apoptotic protein Bcl-xl was coexpressed in our experiments. The expression of Bcl-xl had little or no effect on neuronal polarity, and on axonal or dendrite development (Supplemental Fig. 12) (Gaudillière et al. 2004; Konishi et al. 2004). In addition, FOXO knockdown impaired neuronal polarity in the presence or absence of Bcl-xl (Supplemental Fig. 12). 
Primary hippocampal cultures were prepared as described (Brewer et al. 1993). Neurons were plated on poly-D-lysine-coated glass coverslips at a density of 200 neurons per square millimeter in Neurobasal medium containing B27 supplement, $0.5 \mathrm{mM}$ L-glutamine, $12.5 \mu \mathrm{M}$ glutamate, and penicillin/streptomycin. Transfections were performed 12-16 h after plating using a modified calcium phosphate method (Konishi et al. 2002). A GFP or DsRed2 expression plasmid was cotransfected in all experiments to reveal neuronal morphology. Cells were fixed at the indicated time points and subjected to immunocytochemistry with the GFP (Molecular Probes) or DsRed (BD Biosciences) antibody, together with the MAP2 (Sigma), Tau-1 (Chemicon), Tuj1 (Covance), or MEF2A (Santa Cruz Biotechnologies) antibody, and stained with the DNA-binding dye bisbenzimide (Hoechst 33258).

\section{In vivo electroporation and immunohistochemistry}

In vivo electroporation was performed as described (Konishi et al. 2004). Briefly, P3 Sprague-Dawley rat pups were anesthetized and injected with the indicated plasmids as described (Konishi et al. 2004). After injection, pups received five electric pulses (160 V, 75 msec duration, $950 \mathrm{msec}$ interval) delivered with a CUY-21 square wave electroporator (Protech). Pups were returned to dams and sacrificed 5 or $9 \mathrm{~d}$ later. Transfection efficiency was monitored by GFP fluorescence of dissected cerebella, and positive brains were prepared for cryosectioning. Sections $(20 \mu \mathrm{m}$ or $40 \mu \mathrm{m})$ of P8 or P12 rat cerebella were prepared and subjected to immunohistochemistry with a GFP antibody (Molecular Probes) and an anti-calbindin antibody (Sigma) to label Purkinje cells, and stained with the DNA-binding dye bisbenzimide (Hoechst 33258).

\section{Morphological analysis of cerebellar granule neurons and hippocampal neurons}

To characterize morphology of cerebellar granule neurons or hippocampal neurons, individual images were captured randomly and in a blinded manner on a Nikon eclipse TE2000 epifluorescence microscope using a digital CCD camera (Diagnostic Instruments). Images were imported into Spot Imaging Software (Diagnostic Instruments), and length of neuronal processes was analyzed by tracing. Total length refers to the length of processes, including all its branches added together for a given neuron. To analyze neuronal morphology in vivo, images of individual granule neurons were captured in a blinded manner and measured as above. Calbindin and Hoechst 33258 staining were used to reveal cerebellar cortex anatomy. Cerebellar granule neurons residing in the IGL were selected for morphometry. To study abnormalities in parallel fiber development, the number of parallel fibers and cell bodies present in a specific region of a section were counted in consecutive sections in a blinded manner at 40× magnification as described (Stegmüller et al. 2006).

To analyze neuron polarization, neurons were scored in a blinded manner as polarized or nonpolarized according to reported criteria (Shi et al. 2003). A neuron in which the longest neurite was at least twice as long as the other neurites was considered polarized. Data were collected from three independent experiments, with 50-100 neurons scored per condition per experiment.

A protocol to quantify enrichment of Taul and MAP2 in the longest neurite in hippocampal and cerebellar granule neurons was adapted from Kishi et al. (2005). Images of polarized and nonpolarized neurons were captured as above, and immunofluorescence intensity was quantified using imaging software. Background immunofluorescence intensity of a noncellular area was subtracted from the mean Taul or MAP2 immunofluorescence intensity in each neuronal process to obtain the corrected Taul or MAP2 intensity per process. Tau1 and MAP2 enrichment was defined as the ratio of corrected Tau1 or MAP2 intensity in the longest neurite to the second-longest neurite. Data were collected from two or three independent experiments, with 20-25 neurons per condition per experiment.

\section{Polarity gene expression analyses in neurons}

Granule neurons were transfected at high efficiency by nucleofection with the control U6 or U6/foxo RNAi plasmid. Two days later, RNA was extracted using Trizol according to the manufacturer's instructions, and cDNA was prepared using oligodT primers and SuperScript III reverse transcriptase (Invitrogen) according to manufacturer's protocol. Quantitative PCR was performed with the LightCycler 480 SYBR Green 1 Master Kit on a LightCycler 480 thermocycler (Roche). For all quantitative PCR experiments, gene expression was normalized to GAPDH levels. Specific amplification of target genes was confirmed by agarose gel electrophoresis and calculation of melting temperature of the amplified product. Primer sequences for RT-PCR analyses and details of the method are available on request.

\section{RNAi and rescue constructs}

A DNA template-based method of RNAi was used to express hairpin RNAs targeting the sequence GAGCGTGCCCTACTTC AAGG conserved in all FOXO members (Gaudillière et al. 2002; Lehtinen et al. 2006; Yuan et al. 2008). Rescue constructs were generated by engineering silent mutations for the different FOXO members mutated as follows: FOXO1, GTCCGTCCCGT ACTTTAAGG; FOXO3, GAGCGTCCCGTATTTTAAĀG; and FOXO6, CGTCCCGTATTTCAAGG. Knockdown of individual FOXO family $\bar{m}$ embers was achieved with hairpins targeting the following sequences not conserved in other FOXOs: CAACCTG AGCCTGCTAGAAGA (FOXO1), GGAACTTCACTGGTGCT AAG (FOXO3), and CCATCATCCTCAACGACTTCAT (FOXO6).

\section{ChIP}

ChIP analyses were performed as described (Lehtinen et al. 2006). Briefly, granule neurons were cross-linked with $1 \%$ formaldehyde for $10 \mathrm{~min}$, harvested, and sonicated in ChIP lysis buffer (1\% Triton X-100, 1 mM EDTA, 10 mM Tris- $\mathrm{HCl}$ at $\mathrm{pH} 8.0,200$ $\mathrm{mM} \mathrm{NaCl}, 0.1 \% \mathrm{Na}$-Deoxycholate, $0.1 \%$ SDS, protease inhibitors) to generate chromatin-containing DNA with an average size of 300-1000 nt. A combination of FOXO1, FOXO3, and FOXO6 antibodies (Paik et al. 2007) or rabbit IgG was added to each sample and incubated overnight at $4^{\circ} \mathrm{C}$. To purify the immunocomplexes, $20 \mu \mathrm{L}$ of magnetic Dynabeads (Invitrogen) conjugated to Protein-A were added to the samples and incubated for $1 \mathrm{~h}$ at $4^{\circ} \mathrm{C}$. The beads were washed with lysis buffer, wash buffer (0.1\% Triton X-100, 5 mM EDTA, $30 \mathrm{mM}$ Tris- $\mathrm{HCl}$ at $\mathrm{pH} 8.0,150$ $\mathrm{mM} \mathrm{NaCl}$ ), and TE buffer. The bound protein-DNA immunocomplexes were eluted with $100 \mu \mathrm{L}$ of elution buffer (100 mM $\mathrm{NaHCO}_{3}, 1 \% \mathrm{SDS}, 10 \mathrm{mM} \mathrm{DTT}$ ), and cross-linking was reversed for $4 \mathrm{~h}$ at $65^{\circ} \mathrm{C}$. Next, $250 \mu \mathrm{L}$ of TE, $5 \mu \mathrm{g}$ of glycogen, $100 \mu \mathrm{g}$ of Proteinase K were added to the eluates and incubated for $2 \mathrm{~h}$ at $37^{\circ} \mathrm{C}$. Chromatin DNA was purified by phenol-chloroform extraction and dissolved in $50 \mu \mathrm{L}$ of TE buffer. The purified chromatin DNA was subjected to quantitative PCR with the following primers: Rat GAPDH (forward), GCCTCGTCTCATAGACA AGATGG; Rat GAPDH (reverse), TGCTCCTGCTACTTTAG ACTCCG; Rat Pak1 (forward), GTCTAAAGGTTGCTTCTGT TGC; Rat Pak1 (reverse), GTGACCTCTTCCCTTCATGTTC. 


\section{Luciferase reporter assays}

The upstream 1.4-kb region of the rat Pak1 gene was cloned into the pGL2basic (Promega) vector to generate the Pak1-luciferase reporter gene (Pak1-Luc) using PCR with two primers (5'-CAA CACGCGTCAGCCTGTGAGTGCTGTGTT-3' and 5' -CGACA GATCTGCTG-CAAAGAGCCGGTAATA-3'). Granule neurons transfected $2 \mathrm{~d}$ after plating with a modified calcium phosphate method (Konishi et al. 2002, 2004; Gaudillière et al. 2004) were harvested $36 \mathrm{~h}$ later and subjected to dual-luciferase assays (Promega). In all experiments, neurons were transfected with a Renilla firefly reporter to control for transfection efficiency.

\section{Antibodies}

Antibodies for FOXO1, FOXO3, Pak1, and cleaved caspase 3 were purchased from Abcam, Upstate Biotechnologies, Santa Cruz Biotechnologies, and Cell Signaling Technology, respectively. For Western blots, we used a FOXO6 antibody generated by immunizing rabbits with the FOXO6 peptide AEGSEDSGP ERRATAPA. For ChIP experiments, we used an antibody generated by immunizing rabbits with full-length FOXO6.

\section{Statistics}

Statistical analyses were performed using Statview 5.0.1 (SAS Institute). In experiments in which only two groups were analyzed, comparison of the two groups was done by Student's $t$-test. Pairwise comparison within multiple groups was done by analysis of variance (ANOVA) followed by the Bonferroni post hoc test. All histogram data were obtained from three or more independent experiments and are presented as mean \pm SEM unless otherwise specified. Statistical information and the total number of cells analyzed per experiment are provided in the figure legends.

\section{Acknowledgments}

We thank Constance Cepko, Gabriel Corfas, and David van Vactor for helpful discussions; Marten P. Smidt for the FOXO1-GFP, FOXO3-GFP, and FOXO6-GFP plasmids; Jonathan Chernoff for the Pak1 plasmid; Margareta Nikolic for the Pak1 shRNA; and members of the Bonni laboratory for helpful discussions and critical reading of the manuscript. This work was supported by $\mathrm{NIH}$ grants to A. Bonni (NS041021 and NS051255), A. Brunet (AG026648), the National Science Foundation (L.T.U. and Y.Y.), the Albert J. Ryan Foundation (L.T.U. and Y.Y.) the Edward and Anne Lefler Fellowship (Y.Y.), the Human Frontier Science Program Long-term Fellowship (Y.I.), the Japan Society for the Promotion of Science Fellowship (T.Y.), and the Deutsche Forschungsgemeinschaft (J.S.).

\section{References}

Accili D, Arden KC. 2004. FoxOs at the crossroads of cellular metabolism, differentiation, and transformation. Cell 117: 421-426.

Aizawa H, Hu SC, Bobb K, Balakrishnan K, Ince G, Gurevich I, Cowan M, Ghosh A. 2004. Dendrite development regulated by CREST, a calcium-regulated transcriptional activator. Science 303: 197-202.

Altman J, Bayer S.A. 1997. Development of the cerebellar system: In relation to its evolution, structure, and functions. CRC Press, Boca Raton, FL.

Alvarez B, Martinez AC, Burgering BM, Carrera AC. 2001. Forkhead transcription factors contribute to execution of the mitotic programme in mammals. Nature 413: 744-747.
Arden KC. 2004. FoxO: Linking new signaling pathways. Mol Cell 14: 416-418.

Arias-Romero LE, Chernoff J. 2008. A tale of two Paks. Biol Cell 100: $97-108$.

Arimura N, Kaibuchi K. 2007. Neuronal polarity: From extracellular signals to intracellular mechanisms. Nat Rev Neurosci 8: 194-205.

Barnes AP, Lilley BN, Pan YA, Plummer LJ, Powell AW, Raines AN, Sanes JR, Polleux F. 2007. LKB1 and SAD kinases define a pathway required for the polarization of cortical neurons. Cell 129: 549-563.

Barthel A, Schmoll D, Unterman TG. 2005. FoxO proteins in insulin action and metabolism. Trends Endocrinol Metab 16: 183-189.

Bokoch GM. 2003. Biology of the p21-activated kinases. Annu Rev Biochem 72: 743-781.

Brewer GJ, Torricelli JR, Evege EK, Price PJ. 1993. Optimized survival of hippocampal neurons in B27-supplemented Neurobasal, a new serum-free medium combination. J Neurosci Res 35: 567-576.

Brunet A, Bonni A, Zigmond MJ, Lin MZ, Juo P, Hu LS, Anderson MJ, Arden KC, Blenis J, Greenberg ME. 1999. Akt promotes cell survival by phosphorylating and inhibiting a Forkhead transcription factor. Cell 96: 857-868.

Burgering BM, Kops GJ. 2002. Cell cycle and death control: Long live Forkheads. Trends Biochem Sci 27: 352-360.

Carter ME, Brunet A. 2007. FOXO transcription factors. Curr Biol 17: R113-R114. doi: 10.1016/j.cub.2007.01.008.

Causeret F, Terao M, Jacobs T, Nishimura YV, Yanagawa Y, Obata K, Hoshino M, Nikolic M. 2009. The p21-activated kinase is required for neuronal migration in the cerebral cortex. Cereb Cortex 19: 861-875.

Coffer PJ, Burgering BM. 2004. Forkhead-box transcription factors and their role in the immune system. Nat Rev Immunol 4: 889-899.

Craig AM, Banker G. 1994. Neuronal polarity. Annu Rev Neurosci 17: 267-310.

de Anda FC, Pollarolo G, Da Silva JS, Camoletto PG, Feiguin F, Dotti CG. 2005. Centrosome localization determines neuronal polarity. Nature 436: 704-708.

Edwards DC, Sanders LC, Bokoch GM, Gill GN. 1999. Activation of LIM-kinase by Pak1 couples Rac/Cdc42 GTPase signalling to actin cytoskeletal dynamics. Nat Cell Biol 1: 253-259.

Gaudillière B, Shi Y, Bonni A. 2002. RNA interference reveals a requirement for myocyte enhancer factor $2 \mathrm{~A}$ in activitydependent neuronal survival. J Biol Chem 277: 46442-46446.

Gaudillière B, Konishi Y, de la Iglesia N, Yao G, Bonni A. 2004. A CaMKII-NeuroD signaling pathway specifies dendritic morphogenesis. Neuron 41: 229-241.

Goldberg JL. 2004. Intrinsic neuronal regulation of axon and dendrite growth. Curr Opin Neurobiol 14: 551-557.

Grueber WB, Jan LY, Jan YN. 2003. Different levels of the homeodomain protein cut regulate distinct dendrite branching patterns of Drosophila multidendritic neurons. Cell 112: 805-818.

Hand R, Bortone D, Mattar P, Nguyen L, Heng JI, Guerrier S, Boutt E, Peters E, Barnes AP, Parras C, et al. 2005. Phosphorylation of Neurogenin 2 specifies the migration properties and the dendritic morphology of pyramidal neurons in the neocortex. Neuron 48: 45-62.

Hayashi ML, Choi SY, Rao BS, Jung HY, Lee HK, Zhang D, Chattarji S, Kirkwood A, Tonegawa S. 2004. Altered cortical synaptic morphology and impaired memory consolidation in forebrain-specific dominant-negative PAK transgenic mice. Neuron 42: 773-787. 
Hayashi ML, Rao BS, Seo JS, Choi HS, Dolan BM, Choi SY, Chattarji S, Tonegawa S. 2007. Inhibition of p21-activated kinase rescues symptoms of fragile $\mathrm{X}$ syndrome in mice. Proc Natl Acad Sci 104: 11489-11494.

Hoekman MF, Jacobs FM, Smidt MP, Burbach JP. 2006. Spatial and temporal expression of FoxO transcription factors in the developing and adult murine brain. Gene Expr Patterns 6: 134-140.

Jacobs FM, van der Heide LP, Wijchers PJ, Burbach JP, Hoekman MF, Smidt MP. 2003. FoxO6, a novel member of the FoxO class of transcription factors with distinct shuttling dynamics. J Biol Chem 278: 35959-35967.

Jacobs T, Causeret F, Nishimura YV, Terao M, Norman A, Hoshino M, Nikolic M. 2007. Localized activation of p21activated kinase controls neuronal polarity and morphology. J Neurosci 27: 8604-8615.

Jacobson C, Schnapp B, Banker GA. 2006. A change in the selective translocation of the Kinesin-1 motor domain marks the initial specification of the axon. Neuron 49: 797804.

Jan YN, Jan LY. 2003. The control of dendrite development. Neuron 40: 229-242.

Jiang H, Guo W, Liang X, Rao Y. 2005. Both the establishment and the maintenance of neuronal polarity require active mechanisms: Critical roles of GSK- $3 \beta$ and its upstream regulators. Cell 120: 123-135.

Kandel ER, Schwartz JH, Jessell TM. 2000. Principles of neural science. McGraw-Hill, Health Professions Division, New York.

Kim AH, Bonni A. 2007. Thinking within the D box: Initial identification of Cdh1-APC substrates in the nervous system. Mol Cell Neurosci 34: 281-287.

Kishi M, Pan YA, Crump JG, Sanes JR. 2005. Mammalian SAD kinases are required for neuronal polarization. Science 307: 929-932.

Konishi Y, Lehtinen M, Donovan N, Bonni A. 2002. Cdc2 phosphorylation of BAD links the cell cycle to the cell death machinery. Mol Cell 9: 1005-1016.

Konishi Y, Stegmüller J, Matsuda T, Bonni S, Bonni A. 2004. Cdh1-APC controls axonal growth and patterning in the mammalian brain. Science 303: 1026-1030.

Kumar R, Gururaj AE, Barnes CJ. 2006. p21-activated kinases in cancer. Natl Rev 6: 459-471.

Lehtinen MK, Yuan Z, Boag PR, Yang Y, Villen J, Becker EB, DiBacco S, de la Iglesia N, Gygi S, Blackwell TK, et al. 2006. A conserved MST-FOXO signaling pathway mediates oxidative-stress responses and extends life span. Cell 125: 987-1001.

Nikolic M. 2008. The pak1 kinase: An important regulator of neuronal morphology and function in the developing forebrain. Mol Neurobiol 37: 187-202.

Paik JH, Kollipara R, Chu G, Ji H, Xiao Y, Ding Z, Miao L, Tothova Z, Horner JW, Carrasco DR, et al. 2007. FoxOs are lineage-restricted redundant tumor suppressors and regulate endothelial cell homeostasis. Cell 128: 309-323.

Polleux F, Ince-Dunn G, Ghosh A. 2007. Transcriptional regulation of vertebrate axon guidance and synapse formation. Nat Rev Neurosci 8: 331-340.

Powell SK, Rivas RJ, Rodriguez-Boulan E, Hatten ME. 1997. Development of polarity in cerebellar granule neurons. I Neurobiol 32: 223-236.

Ramón y Cajal S. 1995. Histology of the nervous system of man and vertebrates. Oxford University Press, New York, NY.

Ramos B, Gaudillière B, Bonni A, Gill G. 2007. Transcription factor Sp4 regulates dendritic patterning during cerebellar maturation. Proc Natl Acad Sci 104: 9882-9887.
Schwamborn JC, Puschel AW. 2004. The sequential activity of the GTPases Rap1B and Cdc42 determines neuronal polarity. Nat Neurosci 7: 923-929.

Shalizi A, Gaudillière B, Yuan Z, Stegmüller J, Shirogane T, Ge Q, Tan Y, Schulman B, Harper JW, Bonni A. 2006. A calciumregulated MEF2 sumoylation switch controls postsynaptic differentiation. Science 311: 1012-1017.

Shelly M, Cancedda L, Heilshorn S, Sumbre G, Poo MM. 2007. LKB1/STRAD promotes axon initiation during neuronal polarization. Cell 129: 565-577.

Shi SH, Jan LY, Jan YN. 2003. Hippocampal neuronal polarity specified by spatially localized $\mathrm{mPar} / \mathrm{mPar6}$ and PI 3-kinase activity. Cell 112: 63-75.

Stegmüller J, Bonni A. 2005. Moving past proliferation: New roles for Cdh1-APC in postmitotic neurons. Trends Neurosci 28: $596-601$.

Stegmüller J, Konishi Y, Huynh MA, Yuan Z, Dibacco S, Bonni A. 2006. Cell-intrinsic regulation of axonal morphogenesis by the Cdh1-APC target SnoN. Neuron 50: 389-400.

Tran H, Brunet A, Griffith EC, Greenberg ME. 2003. The many forks in FOXO's road. Sci STKE 2003: RE5. doi: 10.1126/ stke.2003.172.re5.

Van Der Heide LP, Hoekman MF, Smidt MP. 2004. The ins and outs of FoxO shuttling: Mechanisms of FoxO translocation and transcriptional regulation. Biochem J 380: 297-309.

van der Heide LP, Jacobs FM, Burbach JP, Hoekman MM, Smidt MP. 2005. FoxO6 transcriptional activity is regulated by Thr26 and Ser184, independent of nucleo-cytoplasmic shuttling. Biochem J 391: 623-628.

Wittmann T, Bokoch GM, Waterman-Storer CM. 2004. Regulation of microtubule destabilizing activity of Op18/stathmin downstream of Rac1. J Biol Chem 279: 6196-6203.

Yoshimura T, Kawano Y, Arimura N, Kawabata S, Kikuchi A, Kaibuchi K. 2005. GSK-3 $\beta$ regulates phosphorylation of CRMP-2 and neuronal polarity. Cell 120: 137-149.

Yuan Z, Becker EB, Merlo P, Yamada T, DiBacco S, Konishi Y, Schaefer EM, Bonni A. 2008. Activation of FOXO1 by Cdk1 in cycling cells and postmitotic neurons. Science 319: 16651668.

Zhou FQ, Zhou J, Dedhar S, Wu YH, Snider WD. 2004. NGFinduced axon growth is mediated by localized inactivation of GSK- $3 \beta$ and functions of the microtubule plus end binding protein APC. Neuron 42: 897-912. 


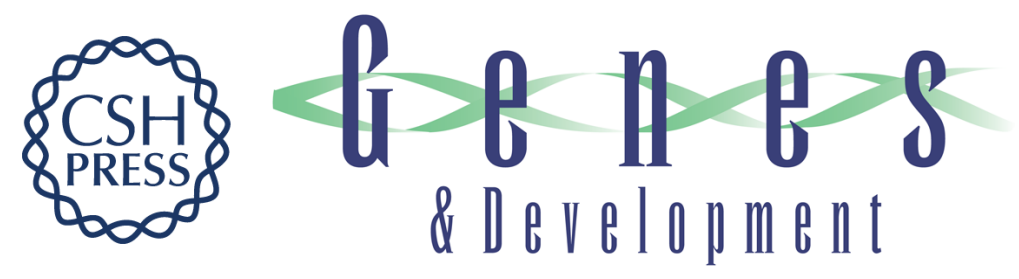

\section{A FOXO-Pak1 transcriptional pathway controls neuronal polarity}

Luis de la Torre-Ubieta, Brice Gaudillière, Yue Yang, et al.

Genes Dev. 2010, 24:

Access the most recent version at doi:10.1101/gad.1880510

Supplemental
Material http://genesdev.cshlp.org/content/suppl/2010/04/12/24.8.799.DC1

References This article cites 55 articles, 11 of which can be accessed free at: http://genesdev.cshlp.org/content/24/8/799.full.html\#ref-list-1

License

Email Alerting Receive free email alerts when new articles cite this article - sign up in the box at the top Service right corner of the article or click here.

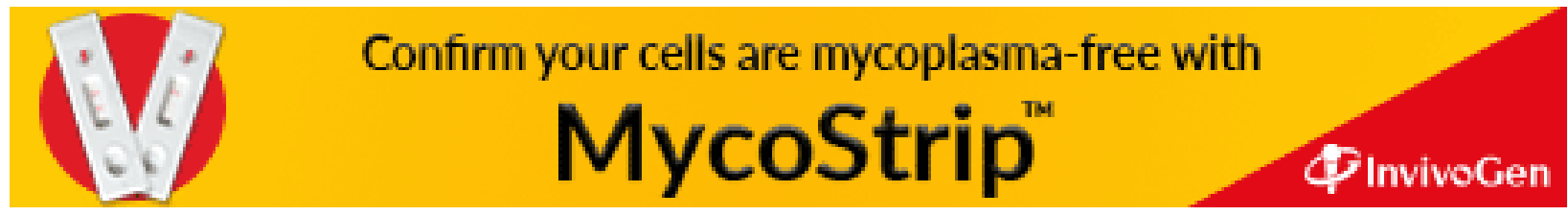

